\title{
Artificially-intelligent robotic space manipulator using fuzzily-architected nonlinear controllers
}

\author{
Shahad S. Ghintab ${ }^{1}$, Zeyad A. Karam ${ }^{2 *}$ and Sami Hasan ${ }^{3}$ \\ Department of Communication Engineering, AL-Mansour University College, Baghdad, Iraq ${ }^{1}$ \\ Department of Systems Engineering, College of Information Engineering, Al-Nahrain University, Baghdad, Iraq ${ }^{2}$ \\ Technical College of Engineering, Albayan University, Baghdad, Iraq ${ }^{3}$
}

Received: 06-November-2021; Revised: 21-February-2022; Accepted: 24-February-2022

(C)2022 Shahad S. Ghintab et al. This is an open access article distributed under the Creative Commons Attribution (CC BY) License, which permits unrestricted use, distribution, and reproduction in any medium, provided the original work is properly cited.

\begin{abstract}
To meet the COVID-19 challenges, the fifth digital industrial wave demands an artificially-intelligent control system advances in selective compliance assembly robot arm (SCARA). Since SCARA has an inherently nonlinear dynamic system and friction rejection, this work develops an artificial intelligent (AI) fuzzy-based nonlinear control algorithm (impedance strategy). Consequently, a generalized dynamic five degree of freedom SCARA (5-Dof SCARA) has mathematically been modelled. These nonlinear controllers have been realized as AI-based fuzzy architectures for the position part from the impedance controller; fuzzy logic proportional derivative (PD) control-type one and Fuzzy logic proportional derivative integral (PID) control type two. These AI architectures regulate the position tracking error of the end effectors' forces. That has been tested using procedures based on half- elliptic and full-elliptic trajectories. Comparatively, the test results are tabulated with related existing published results. Both AI-based controllers have been efficiently dealt with robot nonlinear models and friction rejection. However, the proposed Fuzzy logic PD type one controller has been produced a less-optimum response with the inherent system nonlinearities. Thus, an AI fuzzy-based control algorithm has to be optimally developed to resolve the SCARA sluggishness in achieving tasks. Consequently, a fuzzy logic control (FLC) algorithm of a three-dimension membership function (fuzzy type- 2) has been designed to improve the position response of the SCARA redundant robot end-effectors; Fuzzy logic PID type two-controller. The third dimension is dedicated to overcoming uncertain limits in the nonlinear system that leads to a more stable response in the robot end-effector with no oscillation and zero error. The test results of the AI fuzzy-based PID nonlinear controller has been successfully manifested the superiority in performance to be properly updated in the space shuttle remote manipulator system. Where the large enchantment in position trajectory reached by FLC type-1 PD controller is $93.166 \%$ and $25 \%$ in $X$ and $Y$ axis respectively. The large enhancement by the FLC type-2 PID controller is $95.501 \%$ and $31.250 \%$ in $X$ and $Y$-axis respectively.
\end{abstract}

\section{Keywords}

5-Dof SCARA robot, PID, PD, Impedance controller, Position controller, Fuzzy type-1 controller, Fuzzy type-2 controller, AI fuzzy nonlinear controller, Space shuttle.

\section{Introduction}

The controlling of a selective compliance assembly robot arm (SCARA) manipulator in the space shuttle application is so complicated due to the targeted tasks' accuracy and precision [1]. Figure 1, depicted a typical SCARA. The SCARA robot manipulators are used extensively in the assembly of electronic components and small and medium-sized mechanical devices [2]. SCARA robots are widely used in assembly manufacturing processes.

*Author for correspondence
In general, the SCARA manipulator usually maintains exact moving using a proportional derivative integral (PID) compensator [3]. The application of SCARA redundant manipulator was proposed as redundant sensors on each joint of the space shuttle remote manipulator system (RMS) [4]. Moreover, SCARA of five degrees of freedom (5DOF) has the flexibility and versatility to obtain a wide range of hazardous and critical applications of underwater, and radioactive workplaces. The one DOF SCARA robot with three revolute was first introduced by Hiroshi Makino in 1979 and has become popular in advanced space and critical 
environment applications. Currently, SCARA is a multi-DOF, time-varying, highly nonlinear, and strong coupling system. Consequently, the model has to be developed based on kinematics modelling [5].

SCARA robot is one of the challenging issues in automated industries. SCARA robots are configured for gripping and handling processes in industries. New technology studies improved the performance of the control strategy of the SCARA robot which can replace human action in hazardous, complicated, and multi-tasks [6]. The improvement of this robot controller was considered by researchers in the last two decades. Because of the nonlinearities and complex physical structure, modelling and control are the main problems for SCARA robots. Generally, various kinds of robot controllers give a wide range of performances for robot motion. Most controllers applied the SCARA robot such as PID, fuzzy logic controller (FLC), and also the combination of the two controllers [6]. Consequently, in this work, the 5-DoF SCARA robot dynamic model has been driven. Where the nonlinear dynamic model has been tested under specific affected force on gripper under variation in carried weight in each link.

The main contribution of this paper is to develop artificial intelligence (AI) fuzzy-based nonlinear controller into the force position architecture to control the SCARA robot position trajectory in minimized error in the presence of external disturbances.

The rest of this paper is organized as follows: Literature review has been discussed in section 2 . Section 3 presents the SCARA robot model and the control methodology. Section 4 presents the results and their analysis. Section 5 discusses the results and, finally, section 6 summarizes the work as a conclusion.

\section{Literature review}

Although there is no dearth of existing research works in SCARA control systems, none of them have achieved a comprehensive solution of AI fuzzy-based nonlinear controller. A robust controller was proposed for a SCARA robot using quantitative feedback theory (QFT) [7]. A 5-DOF SCARA redundant manipulator model was tested using fuzzy nonlinear controllers [8]. An adaptive trajectory tracking algorithm was investigated using a sliding mode control (SMC) to subject disturbances and parametric uncertainties [9]. Mamdani-based fuzzy controller was established based on the Lagrange
Euler dynamic model [10]. A various research control projects have utilized conventional and AI fuzzy-based control algorithms to optimize the control action [11-13]. The AI fuzzy-based control algorithms may efficiently improve using a novel fuzzy search tree [14] or fuzzy artificial neural network (FANN), [15]. Some powerful fractional ordered PID (FOPID) controllers are presented for such nonlinear systems and organized to solve multiinput multi-output models [16]. Soriano et al. developed in 2021 a sliding mode control (SMC) algorithm to optimize the energy consumption in the position tracking problem for two degrees of freedom SCARA robot. The gains of the sliding mode controller were refined using the Bat algorithm to save energy by minimizing the forces in actuators [17].

In 2022, a robust control method was proposed for a time-varying and nonlinear system by Zhen, et al. [18] to reduce the errors in the tracking process with the uncertainties of the parameters that appeared in the mechanical system at external disturbance. The thorough study of the above literature shows some errors in mathematical modeling of the redundant SCARA robot based on Euler -Lagrangian method. That motivates this paper's author to re-derive proper mathematical modeling of the redundant SCARA robot under the same conditions as presented in the above literature. Where the results presented in some works cannot be reproduced using the redundant SCARA robot based on Euler-Lagrangian method model. Additionally, the developed controllers were applied up to the full limits, but still, there is a range of errors in position response that cannot be covered according to many uncertainties by the design. So, the author's objective is to

- Derive an accurate mathematical model,

- Apply a control strategy to minimize the error.

- Develop an impedance control strategy (forceposition) based on fuzzy type one and two structures.

The AI fuzzy-based nonlinear impedance control has been vetted to govern the position of the redundant SCARA robot manipulator. This fuzzy controller forcefully moves the end-effector in the desired direction during load-carrying variation and applies forces on the end effector at motion disturbances.

\section{Method}

3.1SCARA robot model

The mechanical structure of the redundant SCARA robot manipulator is presented in Figure 1. This 
structure has five degrees of freedom in the $\mathrm{x}, \mathrm{y}$, and $\mathrm{z}$ directions, in addition to two extra redundant degrees of freedom. That represents the $x-y$ motion, the prism motion along the $\mathrm{z}$-direction, and the redundancy in the rotational motion respectively.

Figurel shows the localization of the axes as coordinates for the robot joints, in addition to presenting the location of the centroid. Where $\mathrm{q}_{1}, \mathrm{q}_{2}$, $\mathrm{q}_{3}, \mathrm{q}_{4}, \mathrm{q}_{5}$ and $\mathrm{l}_{1}, \mathrm{l}_{2}, \mathrm{l}_{3}, \mathrm{l}_{4}, \mathrm{l}_{5}$, represent the generalized coordinates and used links lengths of 1st, 2nd, 3rd, 4 th, and 5th, respectively. And the lengths; 1c2, lc3, and lc4 as depicted in Figure 1.

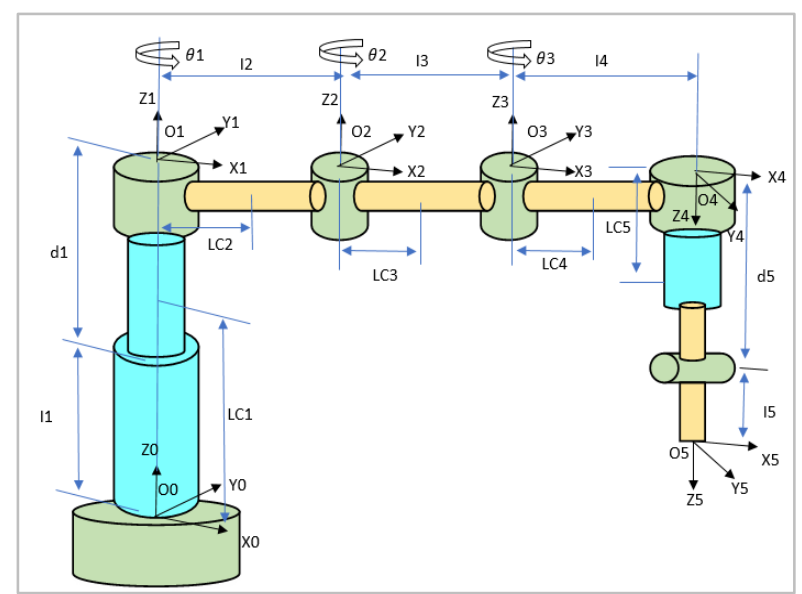

Figure 1 SCARA Arm with two redundant degrees

At first the kinematic of the robot will be derived based on the Denavit-hartenberg (D-H) method [3]. This method is based on calculated parameters illustrated in Table 1 that are used in robot dynamic equation derivation. Equation 1 expresses the (D-H) representation and Equation 2 formulates the $(\mathrm{D}-\mathrm{H})$ representation by acronyms.

Table 1 The SCARA robot-derived D-H parameters

\begin{tabular}{lllll}
\hline Joint & $\emptyset_{\boldsymbol{i}}{ }^{\circ}$ & ${ }^{\circ} \boldsymbol{\alpha}_{\boldsymbol{i}}$ & $\boldsymbol{d}_{\boldsymbol{i}}(\mathbf{m})$ & $\boldsymbol{a}_{\boldsymbol{i}}(\mathbf{m})$ \\
\hline 1 & 0 & 0 & $l_{1}+d_{1}$ & 0 \\
\hline 3 & $\emptyset_{2}$ & 0 & 0 & $l_{2}$ \\
\hline 4 & $\emptyset_{3}$ & 0 & 0 & $l_{3}$ \\
\hline 5 & $\emptyset_{4}$ & 180 & 0 & $l_{4}$ \\
\hline
\end{tabular}

$$
{ }^{i-1} T_{i}=\left[\begin{array}{cccc}
\operatorname{Cos} \theta_{i} & -\operatorname{Cos} \alpha_{i} \operatorname{Sin} \theta_{i} & \operatorname{Sin} \alpha_{i} \operatorname{Sin} \theta_{i} & a_{i} \operatorname{Cos} \theta_{i} \\
\operatorname{Sin} \theta_{i} & \operatorname{Cos} \alpha_{i} \operatorname{Cos} \theta_{i} & -\operatorname{Sin} \alpha_{i} \operatorname{Cos} \theta_{i} & a_{i} \operatorname{Sin} \theta_{i} \\
0 & \operatorname{Sin} \alpha_{i} & \operatorname{Cos} \alpha_{i} & d_{i} \\
0 & 0 & 0 & 1
\end{array}\right]
$$

$$
{ }^{4} T_{0}=\left[\begin{array}{cccc}
C_{234} & S_{234} & 0 & l_{2} C_{2}+l_{3} C_{23}+l_{4} C_{234} \\
S_{234} & -C_{234} & 0 & l_{2} S_{2}+l_{3} S_{23}+l_{4} S_{234} \\
0 & 0 & -1 & l_{1}+d_{1}-l_{5}-d_{5} \\
0 & 0 & 0 & 1
\end{array}\right]
$$

Where, $\quad S_{2}=\sin \theta_{2}, \quad S_{23}=\sin \left(\theta_{2}+\theta_{3}\right), \quad S_{234}=$ $\sin \left(\theta_{2}+\theta_{3}+\theta_{4}\right), C_{2}=\cos \theta_{2}, C_{23}=\cos \left(\theta_{2}+\theta_{3}\right)$, and $C_{234}=$ $\cos \left(\theta_{2}+\theta_{3}+\theta_{4}\right)$.

Form Equation 1, the forward kinematic equation for the end effectors will be:

$$
\begin{aligned}
& P_{x}=l_{2} C_{2}+l_{3} C_{23}+l_{4} C_{234} \\
& P_{y}=l_{2} S_{2}+l_{3} S_{23}+l_{4} S_{234} \\
& P_{z}=l_{1}+d_{1}-l_{5}-d_{5}
\end{aligned}
$$

Equations 1, 2 and 3 represent the end-effector position in the $\mathrm{X}, \mathrm{Y}$, and $\mathrm{Z}$ axes respectively. The second part of the SCARA redundant robot is dynamic modeling. The robot manipulator's kinetic and potential energy is first determined as the Lagrangian of Equation 6 to be substituted in the Lagrange-Euler of Equation 7.

$L(q, \dot{q})=K(q, \dot{q})-U(q)$

$\tau=\frac{d}{d t}\left(\frac{\partial L(q, \dot{q})}{\partial \dot{q}}\right)-\frac{\partial L(q, \dot{q})}{\partial q}$

Where, L; the Lagrangian function symbol, $\mathrm{K}$; the Kinetic energy, U; the potential energy, q; the generalized coordinates vector for robot joints, $\dot{q}$; and $\tau$; generalized velocity and force vectors that included for each joint the forces and torques.

The dynamic model of the robot manipulator with the number of joints can be expressed by Equation 8 [3]:

$\tau=M(q) \ddot{q}+C(q, \dot{q})+G(q)+F(\dot{q})$

Where, $\mathrm{M}$; the inertia matrix in the $\mathrm{n} \times \mathrm{n}$ dimension, $\mathrm{C}$; the Centrifugal and Coriolis forces vector in the $\mathrm{n} \times 1$ dimension, $\mathrm{G}$; the Gravitational force vector in the $\mathrm{n} \times 1$ dimension, $\ddot{q}$; the acceleration of the robot joints vector in the $n \times 1$ dimension, $F$; the vector of the friction forces in the $n \times 1$ dimension. Hence, using Equation 6, Equation 7, and Equation 8, then, the redundant robotic manipulator dynamic model may be expressed as:

$$
\begin{aligned}
& M_{12}=\mathrm{M}_{13}=\mathrm{M}_{14}=\mathrm{M}_{25}=\mathrm{M}_{35}=\mathrm{M}_{45}=0, \\
& \text { and } M_{21}=\mathrm{M}_{12} ; \mathrm{M}_{31}=\mathrm{M}_{13} ; \mathrm{M}_{41}=\mathrm{M}_{14} ; \mathrm{M}_{52} \\
& M=\mathrm{M}_{25} ; \mathrm{M}_{53}=\mathrm{M}_{35} ; \mathrm{M}_{54}=\mathrm{M}_{45} \\
& M=\left[\begin{array}{ccccc}
M_{11} & 0 & 0 & 0 & M_{15} \\
0 & M_{22} & M_{23} & M_{24} & 0 \\
0 & M_{32} & M_{33} & M_{34} & 0 \\
0 & M_{42} & M_{43} & M_{44} & 0 \\
M_{51} & 0 & 0 & 0 & M_{55}
\end{array}\right]
\end{aligned}
$$


Equation 9 represents the inertia matrix.

Let,

$s_{3}=\sin \theta_{3}, s_{4}=\sin \theta_{4}, c_{2}=\cos \theta_{2}, c_{3}=\cos \theta_{3}$, $c_{4}=\cos \theta_{4}, s_{34}=\sin \left(\theta_{3}+\theta_{4}\right), c_{34}=\cos \left(\theta_{3}+\theta_{4}\right)$,

$\mathrm{m}_{1}, \mathrm{~m}_{2}, \mathrm{~m}_{3}, \mathrm{~m}_{4}$, and $\mathrm{m}_{5}$; the masses of the five robot links. $\mathrm{I}_{2 \mathrm{zz}}, \mathrm{I}_{3 \mathrm{zz}}$, and $\mathrm{I}_{4 \mathrm{zz}}$; the Inertia moments for the $2 \mathrm{nd}, 3^{\text {rd }}$, and 4 th links respectively concerning the first $\mathrm{z}$-axis of the 1 st joint. Furtherly, the lengths; $1_{1}, 1_{2}, 1_{3}$, $1_{4}, 1_{5}, 1_{\mathrm{c} 2}, 1_{\mathrm{c} 3}$, and $1_{\mathrm{c} 4}$ are depicted in Figure1.

Equations 10 till Equation 18 specifies the terms of each element from the inertia matrix based on the Euler- Lagrange method.

$M_{11}=\mathrm{m}_{1}+\mathrm{m}_{2}+\mathrm{m}_{3}+\mathrm{m}_{4}+\mathrm{m}_{5}$
$M_{15}=-\mathrm{m}_{5}$
$M_{22}=\left(\mathrm{lc}_{2}{ }^{2} m_{2}\right)+\left(\left(l_{2}{ }^{2}+\mathrm{lc}_{3}{ }^{2}+2 \mathrm{l}_{2} \mathrm{lc}_{3} C_{3}\right) m_{3}\right)+$
$\left(\left(l_{2}{ }^{2}+\mathrm{l}_{3}{ }^{2}+\mathrm{lc}_{4}{ }^{2}+2 \mathrm{l}_{2} l_{3} C_{3}\right) m_{4}\right)+$
$\left(2\left(l_{3} \mathrm{lc}_{4} C_{4}+\mathrm{l}_{2} \mathrm{lc}_{4} C_{34}\right)^{m}\right)+$
$\left(\left(l_{2}{ }^{2}+\mathrm{l}_{3}{ }^{2}+\mathrm{l}_{4}{ }^{2}+2 \mathrm{l}_{2} l_{3} C_{3}\right) m_{5}\right)+$
$\left(2\left(l_{3} l_{4} C_{4}+\mathrm{l}_{2} l_{4} C_{34}\right) m_{5}\right)+\mathrm{I}_{2 \mathrm{zz}}+\mathrm{I}_{3 \mathrm{zz}}+\mathrm{I}_{4 \mathrm{zz}} ;$
$M_{23}=\left(\left(\mathrm{lc}_{3}{ }^{2}+\mathrm{l}_{2} \mathrm{lc}_{3} C_{3}\right) m_{3}\right)+\left(\left(l_{3}{ }^{2}+\mathrm{lc}_{4}{ }^{2}+\right.\right.$
$\left.\left.\mathrm{l}_{2} l_{3} C_{3}+\left(2 \mathrm{l}_{3} \mathrm{lc}_{4} C_{4}\right)\right) m_{4}\right)+\left(\left(l_{2} \mathrm{lc}_{4} C_{34}\right) m_{4}\right)+$
$\left(l_{2} l_{4} C_{34} m_{5}\right)+\left(\left(l_{3}{ }^{2}+\mathrm{l}_{4}{ }^{2}+\mathrm{l}_{2} l_{3} C_{3}+\right.\right.$
$\left.\left.\left(2 \mathrm{l}_{3} l_{4} C_{4}\right)\right) m_{5}\right)+\mathrm{I}_{3 \mathrm{zz}}+\mathrm{I}_{4 \mathrm{zz}} ;$
$M_{24}=\left(\left(\mathrm{lc}_{4}{ }^{2}+\mathrm{l}_{3} \mathrm{lc}_{4} C_{4}+\mathrm{l}_{2} \mathrm{lc}_{4} C_{34}\right) m_{4}\right)+$
$\left(\left(l_{4}{ }^{2}+\mathrm{l}_{3} l_{4} C_{4}+\mathrm{l}_{2} l_{4} C_{34}\right) m_{5}\right)+\mathrm{I}_{4 \mathrm{zz}}$
$M_{33}=\left(\mathrm{lc}_{3}{ }^{2} m_{3}\right)+\left(\left({ }_{3}{ }^{2}+\mathrm{lc}_{4}{ }^{2}+\right.\right.$
$\left.\left.2 \mathrm{l}_{3} \mathrm{lc}_{4} C_{4}\right) m_{4}\right)+$
$\left(\left(l_{3}{ }^{2}+\mathrm{l}_{4}{ }^{2}+2 \mathrm{l}_{3} l_{4} C_{4}\right) m_{5}\right)+\mathrm{I}_{3 \mathrm{zz}}+\mathrm{I}_{4 \mathrm{zz}} ;$
$M_{34}=\left(\left(\mathrm{lc}_{4}{ }^{2}+\mathrm{l}_{3} \mathrm{lc}_{4} C_{4}\right) m_{4}\right)+$
$\left(\left(l_{4}{ }^{2}+\mathrm{l}_{3} l_{4} C_{4}\right) m_{5}\right)+\mathrm{I}_{4 \mathrm{zz}} ;$
$\mathrm{M}_{44}=\left(\mathrm{lc}_{4}{ }^{2} \mathrm{~m}_{4}\right)+(13)$
$\mathrm{M}_{55}=\mathrm{m}_{5} ;$
$\left.{ }^{2} \mathrm{~m}_{5}\right)+\mathrm{I}_{4 \mathrm{zz}} ;$

By inspection

$\mathrm{M}_{32}=\mathrm{M}_{23} ; \mathrm{M}_{42}=\mathrm{M}_{24} ; \mathrm{M}_{43}=\mathrm{M}_{34} ; \mathrm{M}_{54}=\mathrm{M}_{45}$;

The components of centrifugal and Coriolis forces $\mathrm{C}$ are given in Equation 19, specifically by the terms of the Euler Lagrangian method [1]:

$$
\begin{aligned}
& C_{11}=C_{15}=0, \\
& \begin{aligned}
C_{12}=-\left(l_{2} S_{3} \dot{\theta}_{3}^{2}\right. & \left.+2 l_{2} s_{3} \dot{\theta}_{2} \dot{\theta}_{3}\right)\left(l_{c 3} m_{3}+l_{4} m_{4}\right. \\
& \left.+l_{3} m_{5}\right)+\left(l_{2} S_{34} \dot{\theta}_{3}^{2}-2\left(l_{3} S_{4}\right.\right. \\
& \left.\left.+l_{2} S_{34}\right) \dot{\theta}_{3} \dot{\theta}_{4}\right)\left(l_{c 4} m 4+l_{4} m_{5}\right. \\
& +\left(l_{2} S_{34} \dot{\theta}_{2} \dot{\theta}_{3}-\left(\dot{\theta}_{4}^{2}\right.\right. \\
& \left.\left.+2 \dot{\theta}_{2} \dot{\theta}_{4}\right)\left(l_{2} S_{34}\right)\right)\left(l_{c 4} m 4+l_{4} m_{5}\right),
\end{aligned}
\end{aligned}
$$

$$
\begin{gathered}
C_{13}=\left(l_{c 3} m_{3}+l_{3} m_{4}+l_{3} m_{5}\right) l_{2} S_{3} \dot{\theta}_{2}^{2}+\left(l_{2} S_{34} \dot{\theta}_{2}^{2}\right. \\
-\left(2\left(\dot{\theta}_{2}+\dot{\theta}_{3}\right)\right. \\
\left.\left.+\dot{\theta}_{4}\right) l_{3} S_{3} \dot{\theta}_{4}\right)\left(l_{c 4} m 4+l_{4} m_{5}\right), \\
C_{14}=\left(l_{c 4} m_{4}+l_{4} m_{5}\right)\left(l_{3} S_{4}+l_{2} S_{34}\right) \dot{\theta}_{2}^{2}+ \\
\left(l_{2} S_{34} \dot{\theta}_{2}^{2}-\left(2 l_{3} \dot{\theta}_{2} \dot{\theta}_{3}\right)\left(l_{c 4} m 4+l_{4} m_{5}\right)\right.
\end{gathered}
$$

The components of the gravitational force are given by Equation 20 [3]:

$G=\left[\left(m_{1}+m_{2}+m_{3}+m_{4}+m_{5}\right) g, 0,0,0,-m_{5} g\right]^{T}$.

The joint friction consists of Coulomb, Viscous, and Stribeck frictions are specified in Equation 21:

$F=\left[F_{11} F_{12} F_{13} F_{14} F_{15}\right]$;

Where F represents the Forces vector of the friction.

\subsection{Control methodology}

The dynamic model has been implemented, as Figure 2 shows, using the MATLAB Simulink. A type-2 fuzzy set is specified by a three-dimension membership function that is augmented by footprint for uncertainty (FOU). Therefore, type-2 fuzzy sets utilize this propriety as an additional dimension for covering the model uncertainty. Interval type-2 fuzzy logic controllers (IT2FLC) may be utilized for highly handling uncertainty levels in the applications of the mobile robot [12-19]. A typical type-2 fuzzy membership function consists of two membership functions of the type-1 fuzzy system, where the first one is the "upper membership function" (UMF) and "lower membership functions" (LMF). The mathematical representation for FOU can be described by the union between LMF and UMF, the footprint can be described as X Equation 22 [20, 21].

$\operatorname{FOU}(\tilde{X})=\bigcup_{X \in D_{X}} J_{X}$

Where $x$ and $J_{x}$ represent the primary membership of $x,(\tilde{X})$ is an interval type-2 fuzzy membership function and $\mu_{\tilde{X}}(x, u)$ represent the interval type one fuzzy sets as secondary sets, $D_{x}$ is the membership function universe of discourse.

The type- 1 and type-2 fuzzy logic system architecture contains the same mechanism, but with a substitute defuzzifier at the output processor [20]. The IT2FLC diagram may consist of [21];

Fuzzifier

Where the crisps inputs vector $x^{T}=\left(x_{1}, \ldots, x_{p}\right)^{T}$ is entered fuzzification process under the fuzzified block where it is structured into type- 2 fuzzy sets $\tilde{X}$. 


\section{Rules}

The type-1and Type-2 fuzzy system utilizes the IFTHEN conditions as rule architecture, then the consequent for fuzzy type- 2 is represented as follows [21]:

$R^{n}:$ if $\left(x_{1}\right)$ is $\left(\tilde{X}_{1}^{n}\right)$ and ..... and $\left(x_{p}\right)$ is $\left(\tilde{X}_{p}^{n}\right)$ then

(y)is $\left(Y^{n}\right) \quad n=0,1, \ldots \ldots L L \in N$

Where $\tilde{X}_{p}^{n}(i=1, \ldots, P)$ is the input for the type-2 fuzzy system, while the $Y n$ represents the output. For the vector $x=\left(x_{1}, x_{2}, \ldots, x_{p}\right)$ of the $p^{\text {th }}$ inputs; $\mathrm{n}$ are the embedded rules.

\section{Inference}

Type-2 fuzzy systems use the inference engine mechanism to produce the required mapping between the fuzzy sets of the fuzzified input of type- 2 to the defuzzification process after aggregation of the rules (Equation 23-25).

$F^{l}\left(X^{\prime}\right)=\left[\underline{f}^{l}\left(X^{\prime}\right), \bar{f}^{l}\left(X^{\prime}\right)\right]=\left[\underline{f}^{l}, \bar{f}^{l}\right]$

$\underline{f}^{l}\left(X^{\prime}\right)=\mu_{F_{1}^{1}}^{\prime}\left(x_{1}^{\prime}\right) * \ldots \ldots * \mu_{F_{p}^{1}}^{\prime}\left(x_{p}^{\prime}\right)$

$\bar{f}^{l}\left(X^{\prime}\right)=\overline{\overline{\mu_{F_{1}^{1}}^{\prime}}}\left(x_{1}^{\prime}\right) * \ldots \ldots * \overline{\overline{\mu_{F_{p}^{1}}^{\prime}}}\left(x_{p}^{\prime}\right)$

\section{Type-reducer}

The function represents the center of sets, named $Y_{\text {cos }}$ that has mathematical express as [20] (Equation 26 and 27):

$$
Y_{\cos }(X)=\left[y_{1}, y_{r}\right]
$$

$$
\left[y_{1}, y_{r}\right]=\int y^{1} \in\left[y_{l}^{1}, y_{r}^{1}\right] \ldots \int y^{1} \in\left[y_{l}^{M}, M\right]
$$

And the consequent set can be described as (Equation 28):

$$
C_{\tilde{G}^{i}}=\int \theta_{1} \in J_{y_{1}} \ldots \int \theta_{N} \in J_{y_{N}} \frac{1}{\frac{\sum_{i=1}^{N} y_{i} \theta_{i}}{\sum_{i=1}^{N} \theta_{i}}}=\left[y_{l}^{i}, y_{r}^{i}\right]
$$

Equation 28 may be evaluated before the calculation of $Y_{\cos }(x)$. (Equation 29)

$$
y_{l}^{i}=\frac{\sum_{i=1}^{M} f_{l}^{i} y_{l}^{i}}{\sum_{i=1}^{M} f_{l}^{i}} \quad \text { and } \quad y_{r}^{i}=\frac{\sum_{i=1}^{M} f_{r}^{i} y_{r}^{i}}{\sum_{i=1}^{M} f_{r}^{i}}
$$

Karnik and Mendel, as a type reducer mechanism, are used for the type reduction technique.

\section{Defuzzifier}

A type-reducer will be used for obtaining the interval set, which is called $Y_{c o s}$. To denazify this set, an average of $y_{l}$ and $y_{r}$ is founded, then the fuzzified output will be (Equation 30):

$y(X)=\frac{\left(y_{l}+y_{r}\right)}{r}$

Figure 3 depicts the SCARA closed-loop ForcePosition controlled system block diagram

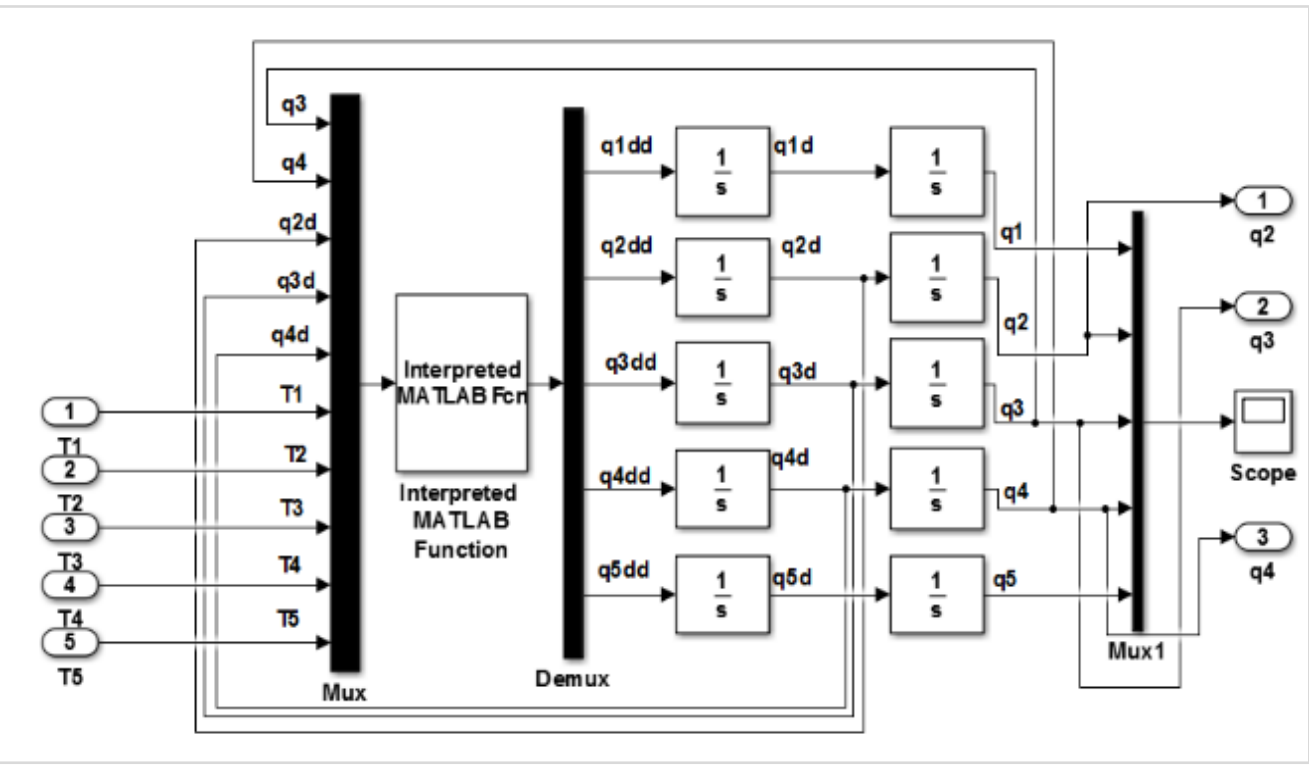

Figure 2 Simulink block of the 5 DoF's redundant robots

Where,

$\mathrm{U} \in \mathrm{R}^{\mathrm{n}}$ is the robot model control law,

$J(q) \in R^{p * n}$ is the matrix of Jacobean,
$\mathrm{K}_{\mathrm{p}}, \mathrm{K}_{\mathrm{v}}$ and $\mathrm{K}_{\mathrm{f}} \in \mathrm{R}^{\mathrm{p} * \mathrm{p}}$ are the force gains for the position, velocity, and matrices, respectively.

$\mathrm{Xd}=\left[\begin{array}{lll}\mathrm{Pd}_{\mathrm{x}} & \mathrm{Pd}_{\mathrm{y}} & \mathrm{Pd}_{\mathrm{z}}\end{array}\right]^{\mathrm{T}}$ are the desired trajectory coordinates in $(\mathrm{m})$, 
$\mathrm{Fd}=\left[\begin{array}{lll}\mathrm{Fd}_{\mathrm{x}} & \mathrm{Fd}_{\mathrm{y}} & \mathrm{Fd}_{\mathrm{z}}\end{array}\right]^{\mathrm{T}}$ are the joint forces at the robot joints in $(\mathrm{N})$,

$\theta_{i}=\left[\theta_{2}, \theta_{3}, \theta_{4}\right]^{\mathrm{T}}$ is the measured angle from each joint in (degree),

$\mathrm{F}=\left[\begin{array}{lll}\mathrm{F}_{\mathrm{x}} & \mathrm{F}_{\mathrm{y}} & \mathrm{F}_{\mathrm{z}}\end{array}\right]^{\mathrm{T}}$ is the generated force vector in $(\mathrm{N})$,

$\mathrm{K}_{\mathrm{f}}=\left[\begin{array}{lll}\mathrm{K}_{\mathrm{fx}} & \mathrm{K}_{\mathrm{by}} & \mathrm{K}_{\mathrm{fz}}\end{array}\right]^{\mathrm{T}}$ are the force gains and $\mathrm{u}=\left[\begin{array}{lll}\mathrm{u}_{\mathrm{x}} & \mathrm{u}_{\mathrm{y}} & \mathrm{u}_{\mathrm{z}}\end{array}\right]^{\mathrm{T}}$ are the outputs of the fuzzy controllers.

The applied three-position controllers in the dynamic SCARA model, as part of the force position control method, are type-2 PD fuzzy logic controllers and then type-2 PID fuzzy logic controllers to deal effectively with nonlinearities in the redundant robot model. The impedance controller Equation is expressed by the following Equations 31 and 32 [22-24].

$u(t)=K_{P}\left(X_{d}-X\right)+K_{v}\left(\dot{X}_{d}-\dot{X}\right)$

$u(t)=\frac{K_{L}}{2}\left(X_{d}-X\right)+K_{V}\left(\dot{X}_{d}-\dot{X}\right)+\frac{K_{A}}{2}\left(X_{d}-\right.$

$X)+K_{I} \int\left(X_{d}-X\right) d t$.

Where

$K_{P}=\frac{K_{L}}{2}+\frac{K_{A}}{2}$
Since the SCARA redundant robot has high nonlinearity, the FLC type-2 is applied to deal directly with this nonlinearity and reach the required tracking enhancements such as enhancing the overshoot, oscillation, and rejection of friction in minimized values. A force position controller includes a three PID controller formed in the structure of FLC type-2 as a position controller for each axis is designed as shown in Figure 3, [15, 21].

The MATLAB Toolbox developed software called Type-2 fuzzy logic (FL). Where the type-2 fuzzy rule for two inputs was chosen; the first one representing the error and the other representing the error change subjected to the linguistic representations as a rule base. Empirically, the upper and lower limits are a big rate of negative (NBUL), medium rate of negative (NMUL), small rate of negative (NSUL), zero rates (ZUL), small rate of positive (PSUL), medium rate of positive (PMUL) and the big rate of positive (PBUL) as summarized in Table 2. The FOU universe of discourses was chosen from 1 to 0.5 with all used membership functions. Seven Gaussian-shaped used as a type for the membership functions in both input and output (Figure 4).

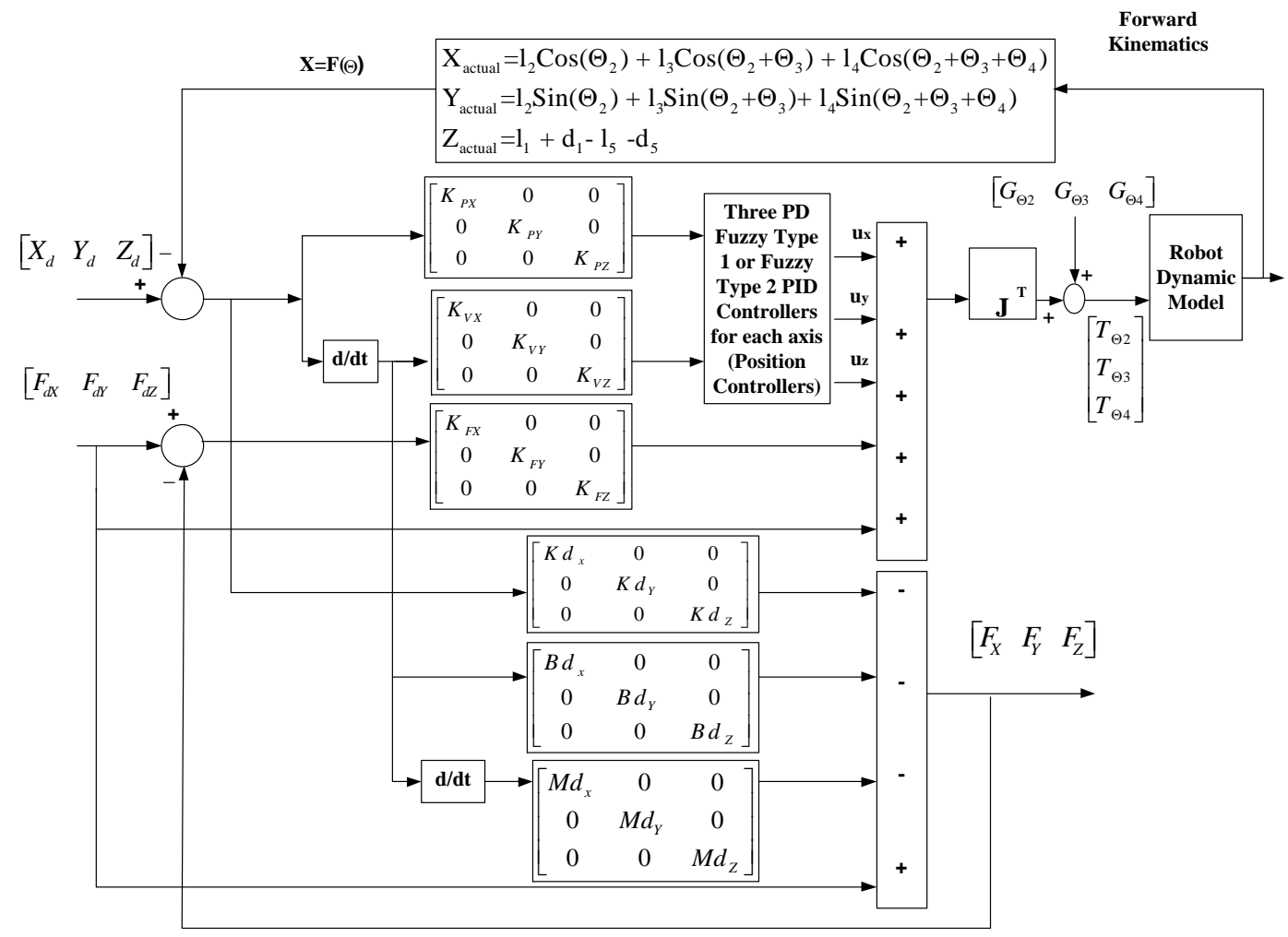

Figure 3 Block diagram of the proposed force position (impedance controller) based on fuzzy type-architecture 

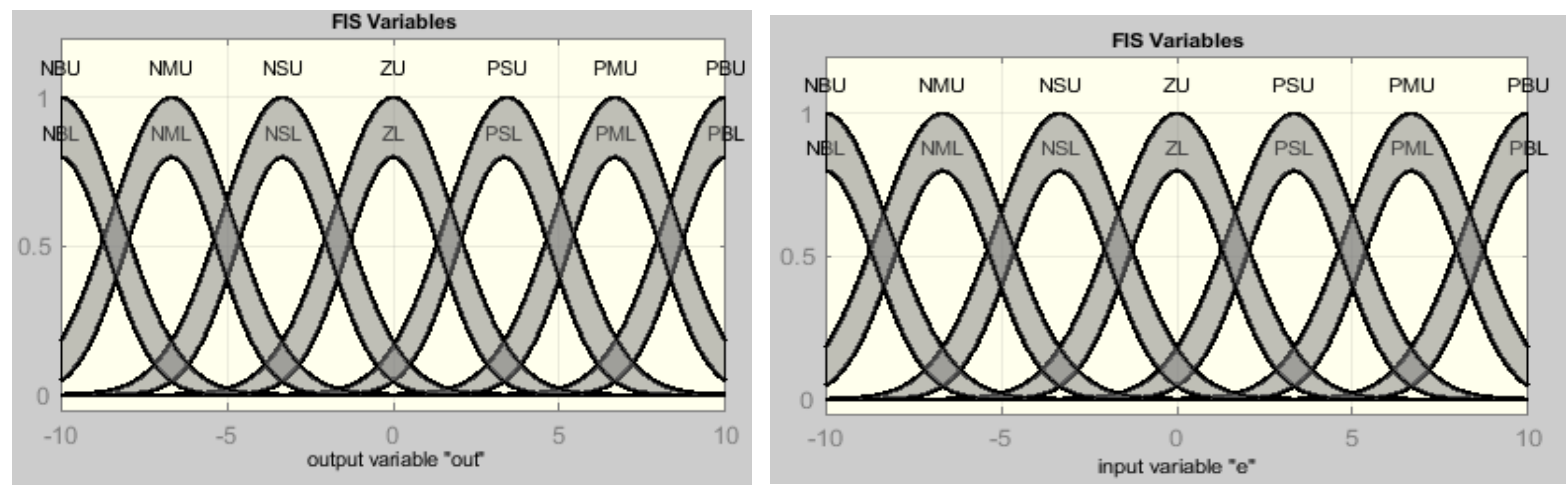

Figure 4 Inputs / output type 2 FLC Membership functions

Table 2 The fuzzy rules of FLC type-2

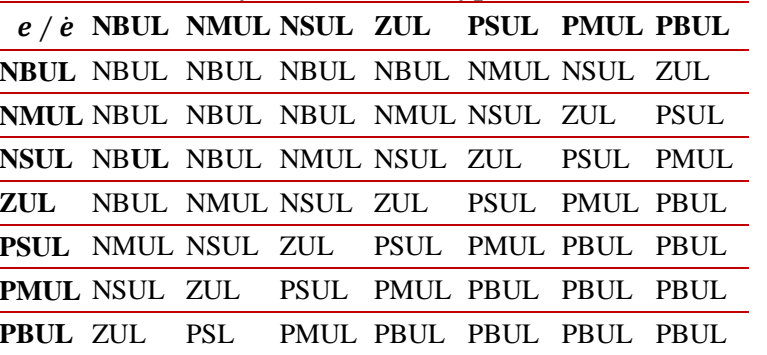

Then the inference engine process infers the entered variables to the equivalent fuzzy set. Then, the output signal is generated by the defuzzification process. Takagi-Sugeno will be used as a method for fuzzy inference with an output range of $-1 /+1$ for negative and positive cases respectively [21]. The input/output scaling factors are defined as proportional- gain $\left(\mathrm{K}_{\mathrm{P}}\right)$, derivative gain $\left(\mathrm{K}_{\mathrm{V}}\right)$, after-derivation gain $\left(\mathrm{K}_{\mathrm{A}}\right)$, and integration gain $\left(\mathrm{K}_{\mathrm{I}}\right)$. The discourse range for $(\mathrm{e}, \dot{e}$, and the output) is taken within $(-10,10)$. The SCARA redundant robot is tested using a force position controller which is incorporate two fuzzy controllers' architecture: the PD FLC type-1 and the PID FLC type-2. Each one of these controllers is tuned manually to the desired minimized position error.

The enhancements in the controlled system are inversely proportional to the error value. The system model parameters are;

$\mathrm{m}_{1}=0.524 \mathrm{~kg}, \mathrm{~m}_{2}=\mathrm{m}_{3}=\mathrm{m}_{4}=1.023 \mathrm{~kg}, \mathrm{~m}_{5}=0.14$ $\mathrm{kg}, \Delta \mathrm{m}_{1}=0.1 \mathrm{~m}_{1} \sin (\mathrm{t}), \Delta \mathrm{m}_{2}=0.1 \mathrm{~m}_{2} \sin (\mathrm{t}), \Delta \mathrm{m}_{3}=$ $0.1 \mathrm{~m}_{3} \sin (\mathrm{t}), \Delta \mathrm{m}_{4}=0.1 \mathrm{~m}_{4} \sin (\mathrm{t}), \Delta \mathrm{m}_{5}=0.1 \mathrm{~m}_{5} \sin (\mathrm{t})$,

$1_{1}=0.524 \mathrm{~m}, \mathrm{l}_{2}=\mathrm{l}_{3}=\mathrm{l}_{4}=0.2 \mathrm{~m}, \mathrm{l}_{5}=0.1_{4} \mathrm{~m}$,

$1_{1 z z}=l_{2 z z}=l_{3 z z}=0.0058 \mathrm{~kg} \cdot \mathrm{m}^{2}$,

$1_{\mathrm{c} 2}=1_{\mathrm{c} 3}=1_{\mathrm{c} 4}=0.0229 \mathrm{~m}$ and

$\mathrm{g}=9.8 \mathrm{~m} / \mathrm{s}^{2}$.

The proposed controllers were individually tested with two reference trajectories for the end effectors to move in the two and three dimensions. The half and full ellipse trajectories are the first and second proposed trajectories respectively. The following designs Equations 33 till Equation 39 represent the desired half ellipse trajectory with maximum end effector applied forces are 1 sin $(t)$ in each axis with the case of FLC type-1 controller and $5 \sin (t)$ with a case of FLC type- 2 controller. $\mathrm{t}_{0}=$ the initial time $=0$; $\mathrm{tf}=$ final simulation time $=20$ second;

$P_{Z}=\mathrm{l}_{1}+\mathrm{d}_{1}-\mathrm{l}_{5}-\mathrm{d}_{5}$

$\mathrm{X}_{1}=0.6 ; \quad \mathrm{X}_{2}=0.4 ; \quad \mathrm{X}_{3}=0 ; \quad \mathrm{X}_{4}=-0.4 ; \quad \mathrm{X}_{5}=-0.6 ; \quad \mathrm{y}_{1}=0$; $\mathrm{y}_{2}=0.3 ; \mathrm{y}_{3}=0.5 ; \mathrm{y}_{4}=0.3 ; \mathrm{y}_{5}=0$;

$\Delta t_{1}=\frac{t_{-} f}{2} ; \Delta t_{2}=\frac{t_{-} f}{2} ;$ If $t \geq 0 \& t \leq \Delta t_{1}, u_{1}=\frac{t}{\Delta t_{1}}$; $P_{x}=2\left(1-u_{1}\right) x_{1}+4 u_{1}\left(1-u_{1}\right) x_{2}+2 u_{1}\left(u_{1}-0\right.$.

5) $x_{3}$

$P_{y}=2\left(1-u_{1}\right) y_{1}+4 u_{1}\left(1-u_{1}\right) y_{2}+2 u_{1}\left(u_{1}-0\right.$.

5) $y_{3}$

Else $t \geq \Delta t_{1} \& t \leq\left(\Delta t_{1}+\Delta t_{2}\right), u_{2}=\frac{t-\Delta t_{1}}{\Delta t_{2}}$ $P_{x}=2\left(1-u_{2}\right)\left(0 \cdot 5-u_{2}\right) x_{3}+4 u_{2}\left(1-u_{2}\right) x_{4}+$ $2 u_{2}\left(u_{2}-0 \cdot 5\right) x_{5}$

$P_{y}=2\left(1-u_{2}\right)\left(0 \cdot 5-u_{2}\right) y_{3}+4 u_{2}\left(1-u_{2}\right) y_{4}+$ $2 u_{2}\left(u_{2}-0 \cdot 5\right) y_{5}$

The second proposed trajectory is the full ellipse trajectory to test the robot end effectors under the same forces applied to each controller type. The following equations represent the designed full ellipse trajectory:

$P_{X}=0.6 \cos (0.314 t)$

$P_{Y}=0.6 \sin (0.314 t)$

\section{Results}

According to the above SCARA model and methodology, the results have two parts. 
4.1Force-position FLC Type-1 PD controller The conventional PID controller may not deal efficiently with the systems and friction rejection. Consequently, a more sophisticated control structure is needed to deal with these nonlinearities and friction rejection. Accordingly, the FLC type-1 PD as a position controller for the force position control method has been proposed. Table 3 Listed the parameters of the force-position strategy-based fuzzy type-1 PD controller. These parameters are established empirically for the half ellipse trajectory. The parameters have been chosen using minimized error estimation in the trajectory tracking process at the presence of the external force's disturbances on the robot end-effector.

Table 3 The Parameters of the force- position strategy based fuzzy type-1 PD controller for the half ellipse trajectory

\begin{tabular}{lllllllll}
\hline Controller & $\mathbf{K}_{\mathbf{P}}$ & $\mathbf{K}_{\mathbf{V}}$ & $\mathbf{K}_{\mathbf{O}}$ & $\mathbf{K}_{\mathbf{d}}$ & $\mathbf{B e}_{\mathbf{d}}$ & $\mathbf{M}_{\mathbf{D}}$ & $\mathbf{K}_{\mathbf{f}}$ & $\mathbf{F}_{\mathbf{D}}$ \\
\hline $\mathbf{X}$-axis & 9 & 15 & 1 & 60 & 150 & 2 & 0.01 & $1 \sin (t)$ \\
\hline Y-axis & 8 & 15 & 1 & 60 & 150 & 2 & 0.01 & $1 \sin (t)$ \\
\hline Z-axis & 10 & 20 & 1 & 60 & 150 & 2 & 0.01 & $1 \sin (t)$ \\
\hline
\end{tabular}

Figure 5 depicts the response of the SCARA redundant robot end-effector subjected to the half ellipse trajectory, the torque control signal in each joint is presented in Figure 6., the active joint angles response and the error in position signal are presented in Figure 7 and Figure 8 respectively. The SCARA robot follows the half-ellipse trajectory, in Figure 5 with a percentage of position error. This error is caused by robot model nonlinearity and the applied external force as disturbances into the robot's end effectors.

Figure 6 profiles the torque performance of the robot DC motors, as actuators; with the maximum nonlinear limit of $\pm 0.3 \mathrm{Nm}$. Figure 7 curves the angles response in each joint motor. Figure 8 plots the error signal founded by the proposed controller for the robot endeffector that is subjected to the half ellipse trajectory with a maximum limit of $0.04 \mathrm{~m}$.

As illustrated in the line graph of Figure 5 the SCARA robot end-effector desired position is represented in the blue line while the actual position is represented in the green line. Overall, from first glance, it is evident that the desired and actual positions vary both marginal and significant starting from negative and moving forward to positive $\mathrm{x}$-axis respectively. The desired position is moving gradually and steadily growing to precisely 0.5 meters reaching the original differs from an actual position which is presented to have a minor decrease around roughly 0.35 , and -0.3 in the $\mathrm{y}$ and $\mathrm{x}$-axis respectively.

Furthermore, the impedance method based on the PD type one fuzzy controller presents a weakness in the process of trajectory tracking and a parameter optimizer is needed to reach the desired error minimization.

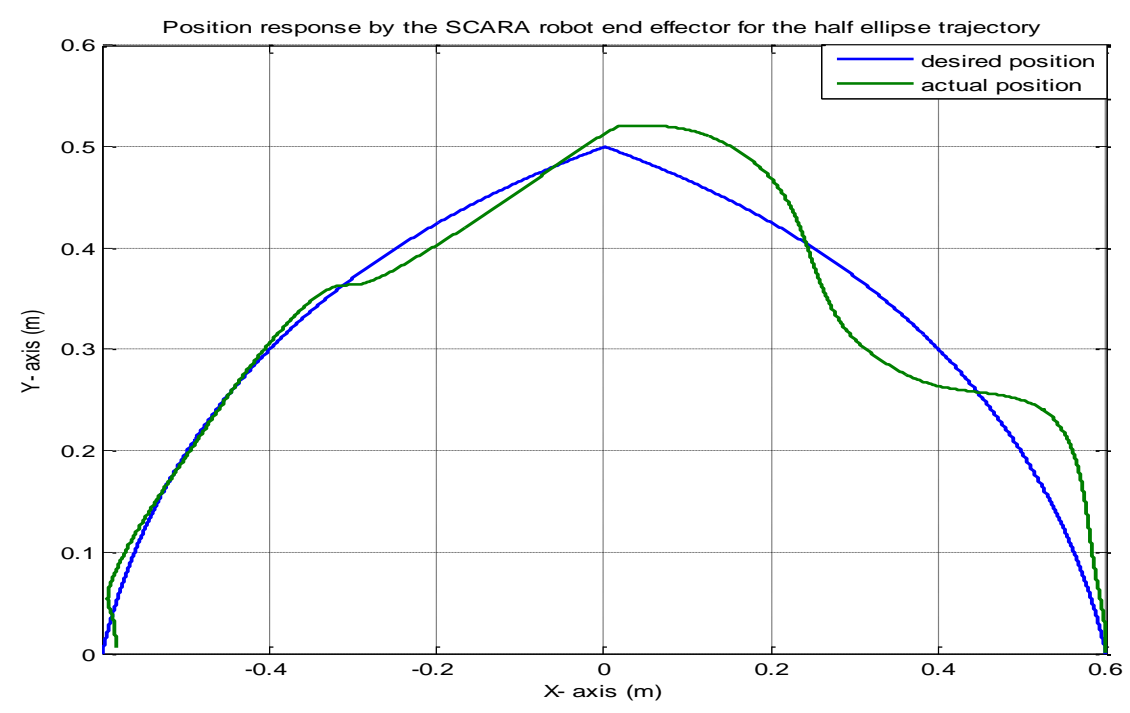

Figure 5 The end effector's position is responsible for the half ellipse trajectory 
Shahad S. Ghintab et al.
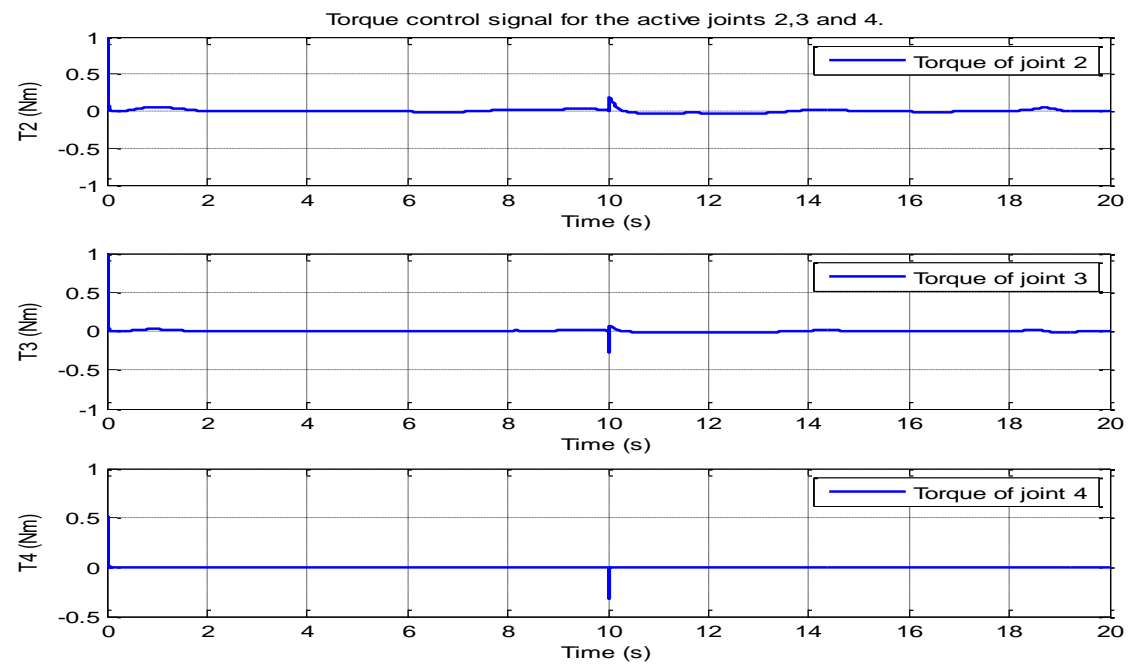

Figure 6 Each active joint has a generated torque control signal

The line graph of Figure 6 depicts the activity of torque control signal for three joints $\mathrm{T}_{2}, \mathrm{~T}_{3}, \mathrm{~T}_{4}$. All are represented in a blue line. The vertical axis represents torque, which increases every 0.5 in unit $\mathrm{NM}$ while the horizontal axis indicates the time, which is presented in a two-second time interval. Cannot dispute both $T_{2}$ and $T_{3}$ in growth in the first second of activity differ to $\mathrm{T}_{4}$ which is presented a plateau activity until reaching 10 seconds of action then undertakes a dip account above $0.5 \mathrm{Nm}$. Like $\mathrm{T}_{4}, \mathrm{~T}_{3}$ also decreases at precisely 10 seconds, roughly just above $0.5 \mathrm{~N} \mathrm{~m}$. Meanwhile, $\mathrm{T}_{2}$ rises roughly below 0.5 $\mathrm{Nm}$. Nevertheless, $\mathrm{T}_{4}$ returns to origin keep a steady and precise path reaching 20 seconds contrary to $T_{3}$ and $\mathrm{T}_{4}$ where both comprise a minor fluctuation in torques around 14 and 18.5 seconds.

The line graph of Figure 7 presents three active joint angle responses for theta 2 , theta 3 , and theta 4 all illustrated in the blue line. The vertical axis represents theta the in-unit degree which changes every 50 degrees. While the horizontal axis indicates the time in seconds. Overall, it is evident that the activity of theta 3 and theta 4 holds an opposite relationship that differs from that of theta 2 which is independent.
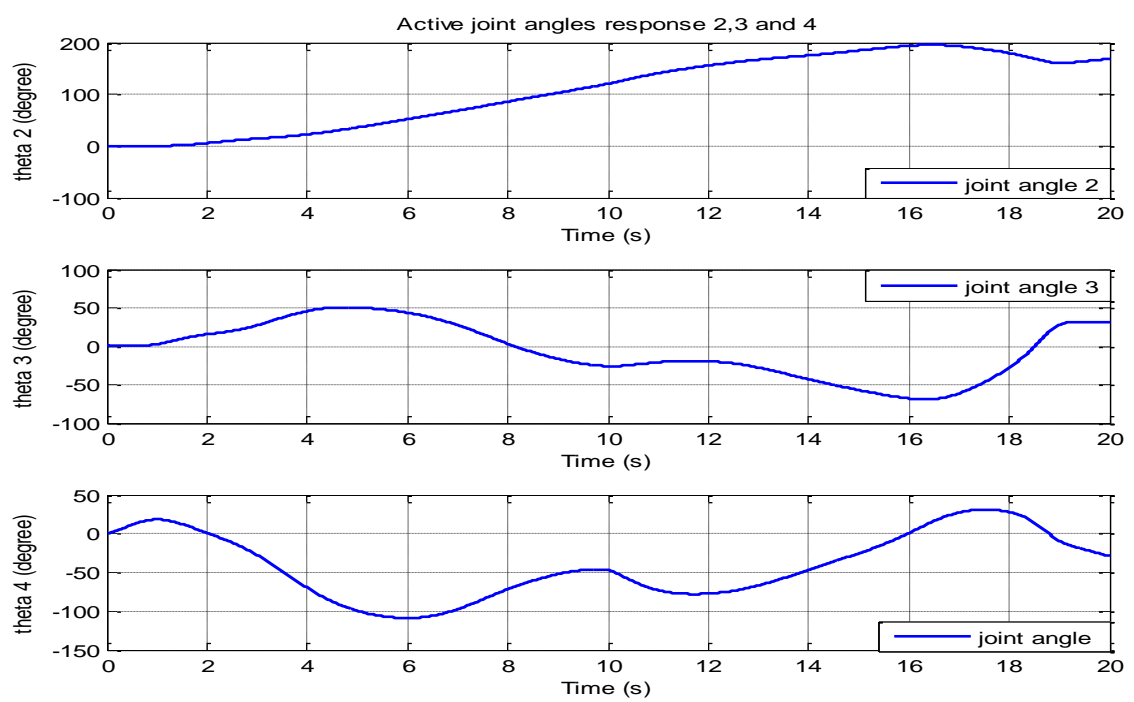

Figure 7 Joint angle response for the active joints 
Cannot dispute that theta 2 gradually increased starting from the first second reaching 100 degrees at precisely 8 seconds, but then experienced a significant increase after 8 seconds making up twice the original quantity marking 200 degrees. However, decreased approximately $25 \%$ in 19 seconds accounting for roughly 170 degrees.

Nevertheless, theta 3 also started at a gradual and steady in growth accounted 50 degrees in 4.5 seconds, but experienced a fall of $80 \%$ original quantity which marks around -25 degrees differs to theta 4 which is presented as a significant in growth starting from the original accounts 25 degrees for the first second then over goes a crucial decrease reaches just above -100 degrees in 5 seconds. Furthermore, increases almost $50 \%$ of the drop in the next 5 seconds reaching -50 degrees. Theta 3 and theta 4 experience a fluctuation between the period from 10 to 20 seconds, but in an opposite wave relationship.

The two-line diagrams of Figure 8 illustrate the activity of half ellipse trajectory. The vertical axes present error of both $\mathrm{y}$ and $\mathrm{x}$-axis in-unit meter and which changes every 0.02 degrees. The horizontal axes indicate the time in unit second changes in 2 second time intervals. In conclusion, the impedance method-based PD fuzzy type one controller gives a high error rate of $0.04 \mathrm{~m}$ that cannot be acceptable in robot position tracking trajectory.
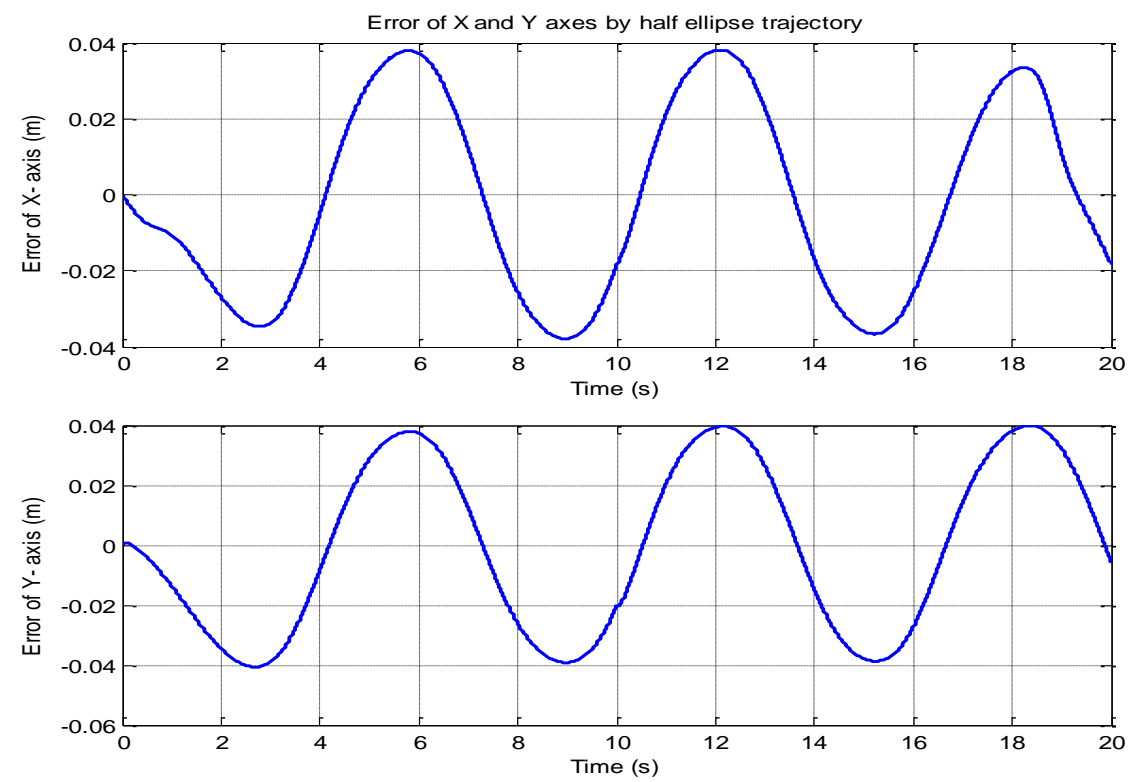

Figure $8 \mathrm{X}$-Y working exes position error signal by the half ellipse trajectory

The response of the end effectors subjected to the full ellipse trajectory is presented in Figures 9, 10,11, and 12.

Table 4 illustrates the parameters of the force position strategy based on fuzzy type-1 PD controllers that are empirically estimated for the full ellipse trajectory. These gains have been chosen using minimized error estimation with no singularity accuracy in the presence of the external force's disturbances at the robot end-effector.

Table 4 The Parameters of the impedance controller, fuzzy type-1 for full ellipse trajectory

\begin{tabular}{lllllllll}
\hline Controller $\mathbf{K}_{\mathbf{P}} \mathbf{K}_{\mathbf{V}}$ & $\mathbf{K}_{\mathbf{0}}$ & $\mathbf{K}_{\mathbf{d}}$ & $\mathbf{B e}_{\mathbf{d}}$ & $\mathbf{M}_{\mathbf{D}}$ & $\mathbf{K}_{\mathbf{f}}$ & $\mathbf{F}_{\mathbf{D}}$ \\
\hline $\mathbf{X}$-axis & 20 & 22 & 1 & 60 & 150 & 2 & 0.01 & $1 \sin (t)$ \\
\hline
\end{tabular}

\begin{tabular}{lllllllll}
\hline Controller & $\mathbf{K}_{\mathbf{P}} \mathbf{K}_{\mathbf{V}}$ & $\mathbf{K}_{\mathbf{O}} \mathbf{K}_{\mathbf{d}}$ & $\mathbf{B e}_{\mathbf{d}}$ & $\mathbf{M}_{\mathbf{D}}$ & $\mathbf{K}_{\mathbf{f}}$ & $\mathbf{F}_{\mathbf{D}}$ \\
\hline $\mathbf{Y}$-axis & 15 & 20 & 1 & 60 & 150 & 2 & 0.01 & $1 \sin (t)$ \\
\hline Z-axis & 10 & 20 & 1 & 60 & 150 & 2 & 0.01 & $1 \sin (t)$
\end{tabular}

The line chart of Figure 9 indicates the marginal changes between the desired and actual position response of SCARA robot, like $y$-axis, $\mathrm{x}$-axis changes every 0.2 in unit meters. The desired position is moving gradually and steadily growing to precisely 0.2 meters, reaching the original differs from the actual position which is presented to have a minor decrease around roughly 0.35 meter and -0.35 meter in $y$ and $x$-axis respectively. The impedance method based on PD type one fuzzy controller showed better response with trajectory tracking process and its application. 


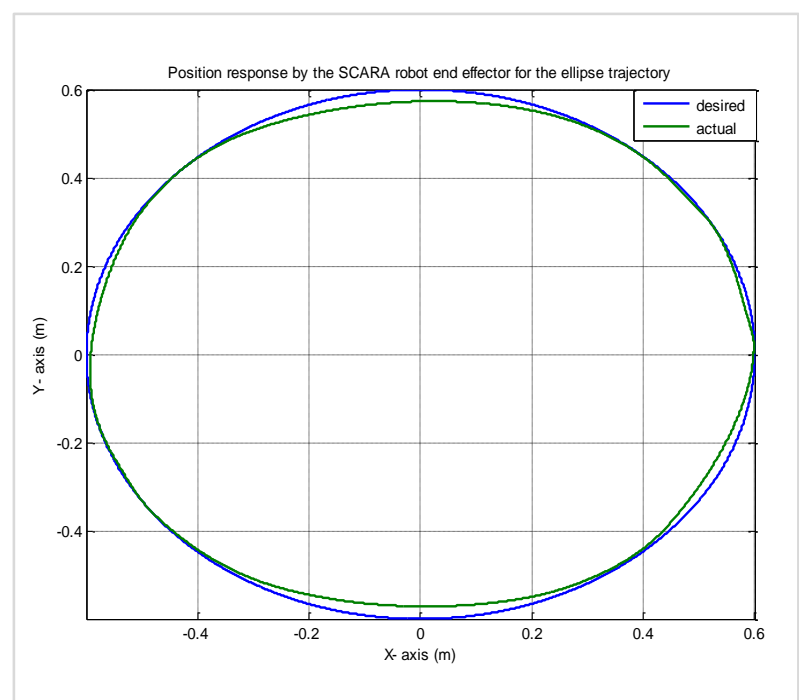

Figure 9 The end effectors' position is responsible for the full ellipse trajectory

The three-line charts of Figure 10 illustrate torque control activity of three joints $\mathrm{T}_{2}, \mathrm{~T}_{3}$, and $\mathrm{T}_{4}$. The horizontal axis indicates the time in unit seconds, which increases every 2 seconds while the vertical axis presents the torque in-unit $\mathrm{Nm}$ which varies in $0.5 \mathrm{Nm}$. The blue line represents the activity of all three joints.

According to the line chart, the activity of both joints $\mathrm{T} 2$ and T3 has a similar pattern of mere fluctuation in the 20 seconds. Moreover, T2 peak activity just above $0.25 \mathrm{Nm}$, then, suddenly declines in the first two seconds. Keeps this pattern for four seconds, but with a fall in quantity. However, this motion change leans to a plateau around the origin for 5 seconds from 4 to 9 seconds, but the variation returns in a much lower intensity. Also, this applies to T3, but with a meager activity. Nevertheless, T4 may be identified that is independent in motion. From the beginning, the torque shows no activity through the whole 29 second period.

The three-line graphs of Figurell depict joint activity presented in angles for theta, 2, theta, 3, and theta 4 the blue line represents the joints. The horizontal indicates the time in unit seconds and the vertical indicates the theta in-unit degree. Notably, one cannot dispute that the motion of theta 2 is steadily increasing from the origin within the same period of both theta 3 and theta 4 . In addition to minimal fluctuation contrary to theta 3 and theta, 4, both very frequently. Nevertheless, theta 4 accounts for roughly 20 degrees in the first 0.5 seconds, but shrinks noticeably in the next 0.5 seconds makeup just below 0 degrees. However, the former peaks remarkably reached approximately 45 degrees in the 4- and 5-seconds time period, but latter falling almost $85 \%$ of its quantity comprises -25 degrees. Furthermore, a pattern of rapid, steep continues from 10 seconds to 18 seconds, which accounts through this period a plunge of -49 degrees and a rise of 20 degrees which differs to theta 2 . At first glance, it is evident that theta 2 from the starting point skyrocketed with mere variations in the first 2 seconds ingrowth 50 degrees, but shortly returned to the steady in growth path reaching 200 degrees in 10 seconds. Moreover, theta 2 underwent a mere dip in 12 and 18 seconds, but this didn't affect its rise. Final accounts for the maximum number of above 300 degrees.

The two-line diagrams of Figure 12 illustrate the activity of full ellipse trajectory, the vertical axes present error of both $\mathrm{y}$ and $\mathrm{x}$-axis in-unit meter and which changes every 0.02 degrees the horizontal axis indicates the time in unit second changes in the 2 second time interval. Overall, the impedance methodbased fuzzy type one controller gives a high error rate of $0.035 \mathrm{~m}$ that cannot be acceptable in robot position tracking trajectory.

By comparing Figure 9 to Figure 5, the SCARA robot follows the full ellipse test curve in a better manner with a noticeably lower error rate under the same working environment. Figure 10 profiles the robot torque performance of actuators (DC motors) with the maximum limit of $\pm 0.27 \mathrm{Nm}$.

Figure 11 and Figure 12 curves the joint motor angles response and the error signal at the full ellipse trajectory with a maximum limit of $0.031 \mathrm{~m}$. The response of the SCARA redundant robot end effectors under the applied force and variable load in each link has been presented in Figure 5 and Figure 9. The proposed FLC type-1PD controller has been produced a less-optimum response with the inherent system nonlinearities. Thus, an AI fuzzy-based control algorithm has to be optimally developed to resolve the SCARA sluggishness in achieving tasks. 

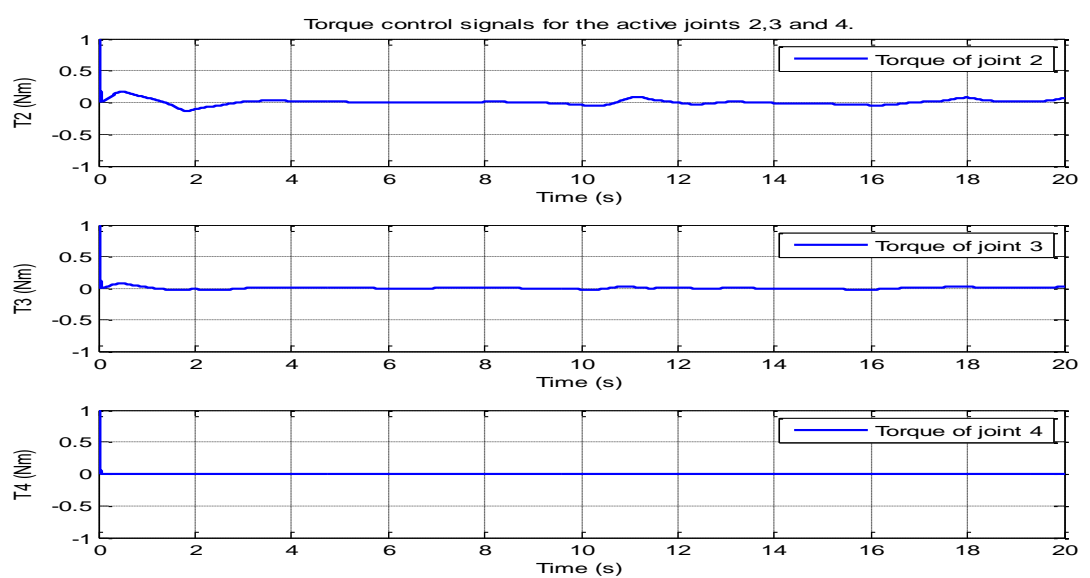

Figure 10 Each active joint has a torque control signal
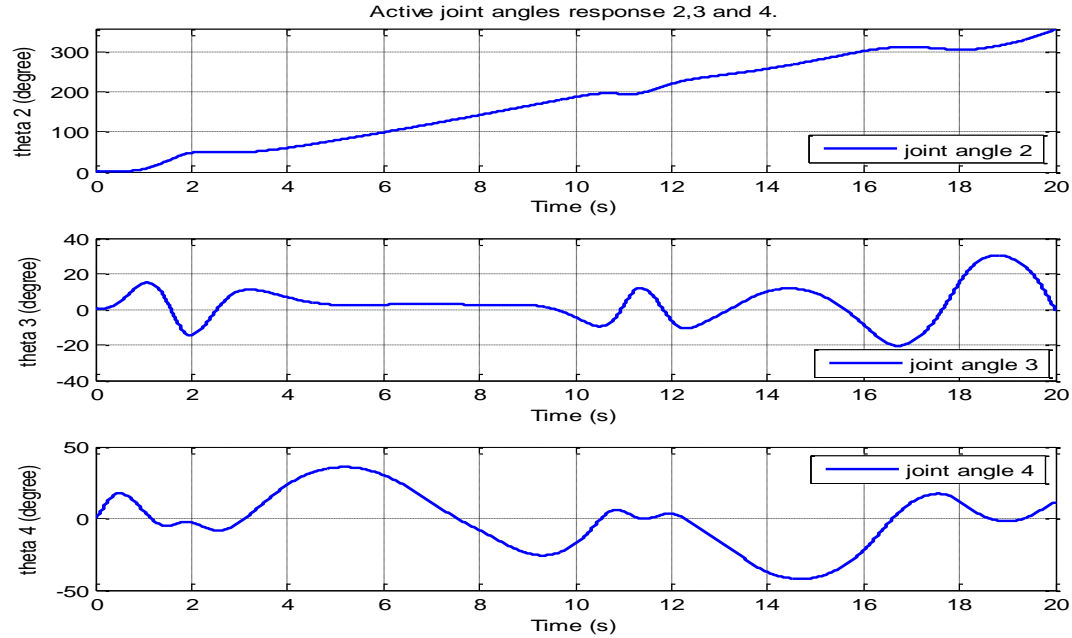

Figure 11 Joint angle response for the active joints
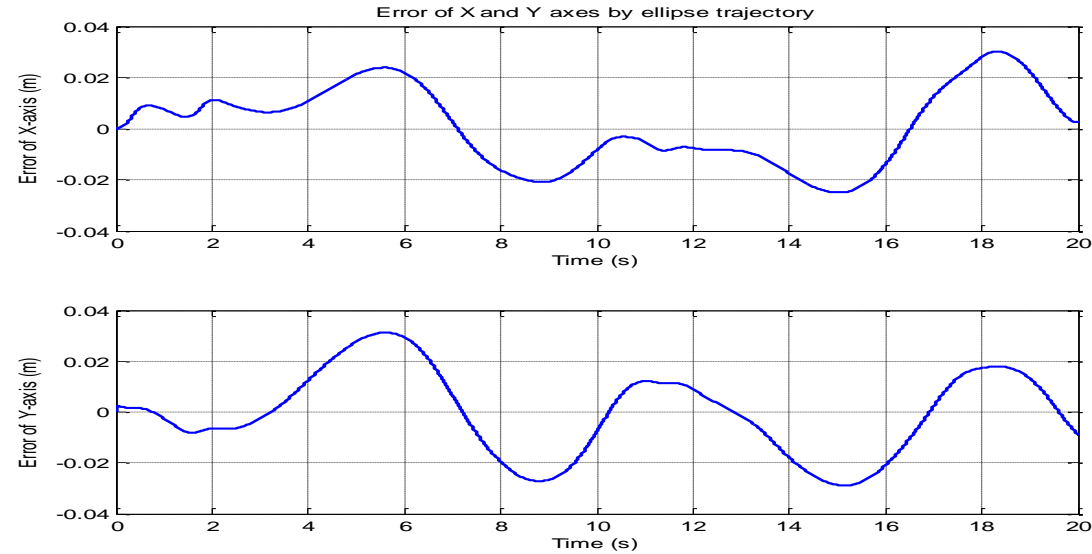

Figure $12 \mathrm{X}-\mathrm{Y}$ working exes position Error signal by the full ellipse trajectory 


\subsection{Force-position FLC type-2 PID controller} Consequently, a fuzzy control algorithm of a threedimension membership function is designed to improve the position response of the SCARA redundant robot end effectors. The third dimension is dedicated to overcoming uncertain limits in the nonlinear system that leads to a more stable response in the robot end-effector with minimum oscillation and error. This new position controller is called the FLC type-2 PID. Table 5 states the force-position strategy based on one fuzzy type-2 PID controller parameters that are experimentally evaluated for the half ellipse trajectory. Where it was chosen based on minimizing error estimation in the trajectory tracking process, while the presence of the external force's disturbances at the robot end-effector.

Table 5 The parameters of the impedance FLC type2 PID controller for the half ellipse test

\begin{tabular}{llllllllll}
\hline Controller & $\mathbf{K}_{\mathbf{P}}$ & $\mathbf{K}_{\mathbf{V}}$ & $\mathbf{K}_{\mathbf{a}}$ & $\mathbf{K}_{\mathbf{I}}$ & $\mathbf{K}_{\mathbf{d}}$ & $\mathbf{B} \mathbf{e}_{\mathbf{d}}$ & $\mathbf{M}_{\mathbf{D}}$ & $\mathbf{K}_{\mathbf{f}}$ & $\mathbf{F}_{\mathbf{D}}$ \\
\hline $\mathbf{X}$-axis & 900 & 25 & 20 & 93 & 60 & 150 & 2 & 0.01 & $5 \sin (t)$ \\
\hline Y-axis & 900 & 25 & 20 & 93 & 60 & 150 & 2 & 0.01 & $5 \sin (t)$ \\
\hline Z-axis & 800 & 36 & 72 & 93 & 60 & 150 & 2 & 0.01 & $5 \sin (t)$
\end{tabular}

Figure 13 represents the response of the SCARA redundant robot end-effector subjected to the half ellipse trajectory. The torque control signal in each joint is presented in Figure 14. The active joint angles response and the error in position signal are presented in Figure 15 and Figure 16 respectively.

The line chart of Figure 13 indicates the half ellipse trajectory directed by end effectors. Where the desired and actual positions are presented in blue and red lines respectively. $\mathrm{Y}$-axis and $\mathrm{X}$-axis change every 0.2 in unit meters. On the whole, there is an emerging difference between the desired and actual positions after the second half of motion differ from the first half which is depicted as the optimum position response. The use of the impedance method based on PID fuzzy type two-controller produced an optimal result that can be applied in the trajectory tracking process due to the footprint ability of the type two fuzzy controllers to deal with systematic uncertainties.

The three-line charts of Figure 14 indicate torque control signals for the active joints of T2, T3, and T4 presented in the blue line. At first glance, T2 and T3 may identify to be similar in the sense of motion waves but differ in magnitude. Whereas, $\mathrm{T} 4$ is independent in motion and quantity. Furthermore, the $\mathrm{T} 2$ signal varies significantly between the period of 0 to 0.9 seconds, which accounts for roughly $1.6 \mathrm{Nm}$ but begins to decline in its quantity reaching its origin at precisely 2 seconds and keeping a plateau zero quantity. Afterward, T2 undergoes a spiral, but a short increase in joint activity of exactly 10 seconds makes up just below $1 \mathrm{Nm}$, shortly after returning to a steady pattern around the origin. The same applies to $\mathrm{T} 3$, but the quantity of the signals shortens around $25 \%$ from $\mathrm{T} 2$. However, T4 signals out take a variation in signals for the first second of the total period. Shortly after returning to the origin and remains at a steady rate of $0 \mathrm{Nm}$ until reaching half the 20 seconds that indicates a significant, but instant decrease in signal, making exactly up to- $0.2 \mathrm{Nm}$.

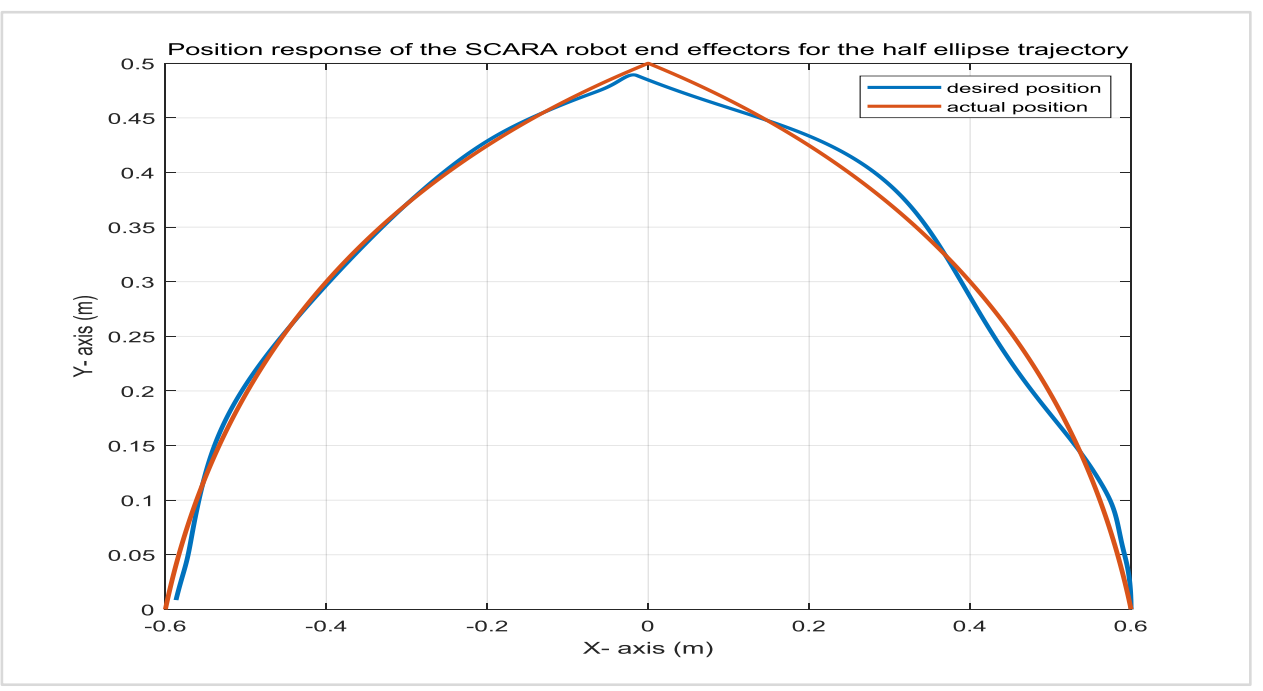

Figure 13 The end effector's position is responsible for the half ellipse trajectory 

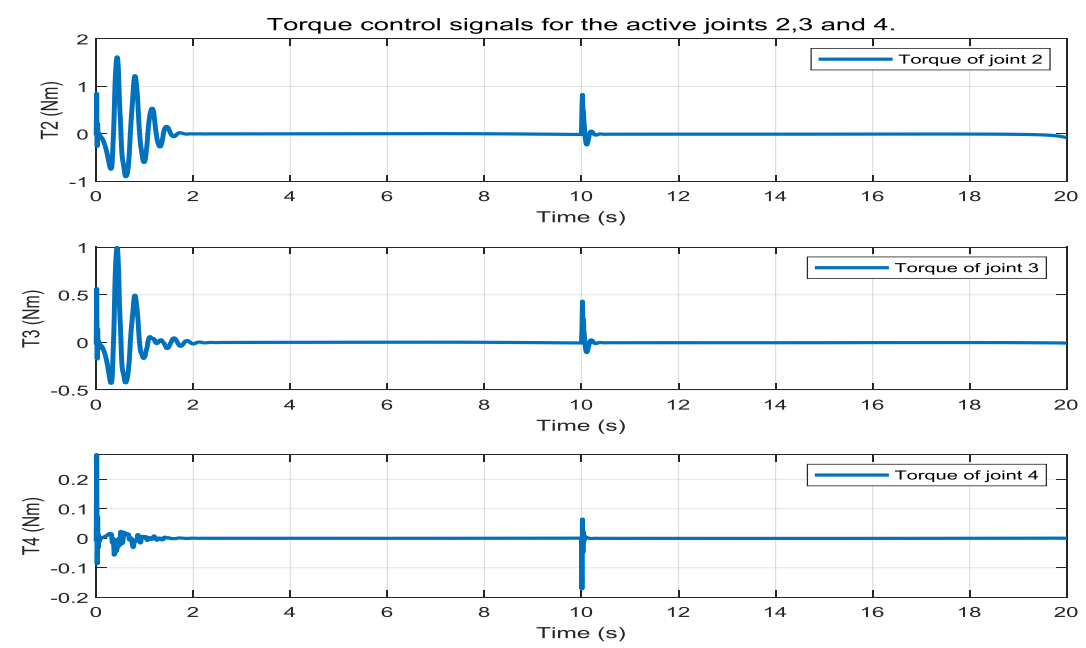

Figure 14 The generated torque control signal in each active joint

The line chart of Figure 15 illustrates three stages of theta responses in three joint angles theta, 2, theta 3, and theta 4 . The blue line represents the angles activity of the joints with the vertical axis representing theta in-unit degrees, while the horizontal axis indicates the time in unit seconds. Figure 15 indicates the drastic changes in all three curves. Nevertheless, the motion, joint of theta 2 is independent on both theta 3 and theta, 4, ending in the most enormous degree compared to the other two activities reaching roughly 200 degrees in 19 seconds. However, theta 3 and theta 4 are evident that both in a significant contrary motion almost collide inactivity. Moreover, theta 3 has a very slow movement that begins precisely 3 seconds, reaches 70 degrees in 8 seconds. While theta 4 skyrockets from the origin reaching just above 50 degrees in the first 4 seconds, but decreasing dramatically to the origin at around 8.5 seconds. Furthermore, theta 3 undergoes a merely low steep in 10 seconds, but shortly increases steadily until reaches 14 seconds where theta 3 motions, gradually decreases to the origin contrary to theta 4 that increases by 14 seconds and reaches 50 degrees.
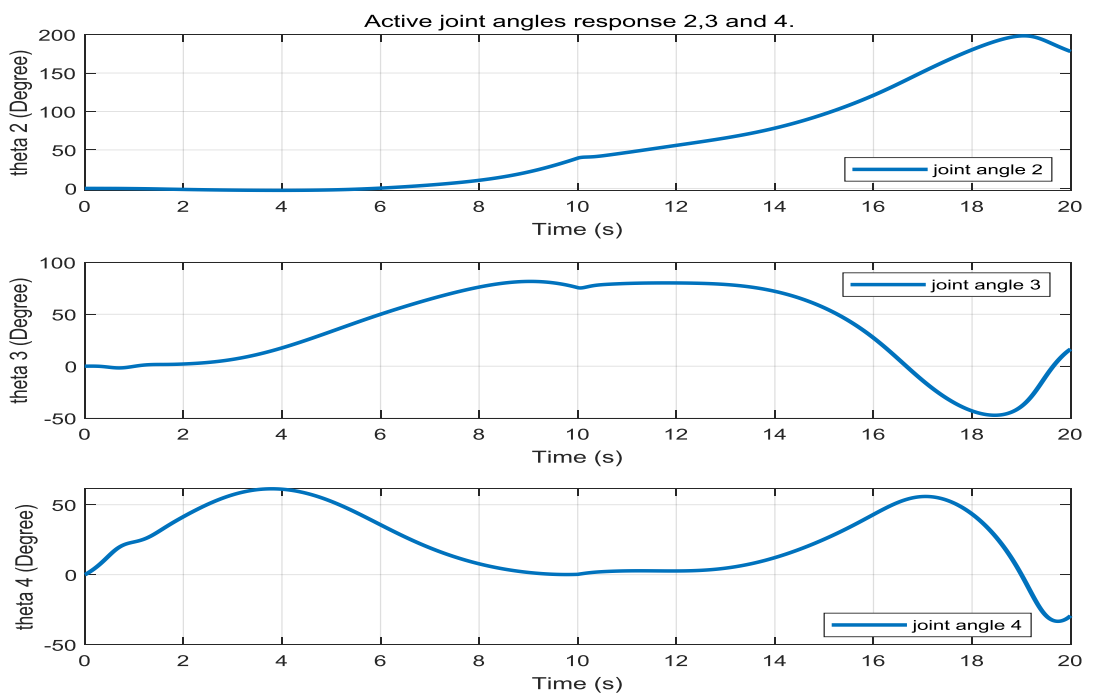

Figure 15 Joint angle response for the active joints 
Both line charts of Figure 16 illustrate the error signals in the working position for half elliptical trajectory. The pattern for both line charts is similarly sinusoidal with minor changes in the first 0.5 and 10 seconds, but the $\mathrm{x}$-axis error signal amplitude is greater by $0.005 \mathrm{~m}$ than that of the $\mathrm{y}$-axis. Where the overall error rate generated by the impedance method based fuzzy type two PID controller is nearly $0.01 \mathrm{~m}$ and it's acceptable.

Figure 13 proves an observable better performance compared to Figure 5. Accordingly, Figure 14 curves the actuator torque performance better than that of Figure 6 with the maximum limit of $\pm 1.6 \mathrm{Nm}$, and, the corresponding joint motor angle response and error signal in Figure 15 and Figure 16 respectively, with the robot end-effector that subjected to the half ellipse trajectory with a maximum limit of $0.016 \mathrm{~m}$. The responses of the end effectors subjected to the full ellipse trajectory are presented in Figure 17, Figure 18, Figure 19, and Figure 20.

Table 6 lists the parameters of the force-position strategy based on fuzzy type-2 PID controllers that have been experimentally evaluated for the full ellipse trajectory. Where the gains have been chosen using minimized error estimation with no singularity accuracy in the presence of the external force's disturbances at the robot end-effector.

Table 6 Parameter of the impedance controller, fuzzy type-2 for full ellipse trajectory

\begin{tabular}{lllllllllllll}
\hline Controller & $\mathbf{K}_{\mathbf{P}}$ & $\mathbf{K}_{\mathbf{V}}$ & $\mathbf{K}_{\mathbf{A}}$ & $\mathbf{K}_{\mathbf{I}}$ & $\mathbf{K}_{\mathbf{d}}$ & $\mathbf{B e}_{\mathbf{d}}$ & $\mathbf{M}_{\mathbf{D}}$ & $\mathbf{K}_{\mathbf{f}}$ & $\mathbf{F}_{\mathbf{D}}$ \\
\hline $\mathbf{X}$-axis & 900 & 27 & 23 & 90 & 60 & 150 & 2 & 0.01 & $5 \sin (t)$ \\
\hline Y-axis & 950 & 25 & 20 & 89 & 60 & 150 & 2 & 0.01 & $5 \sin (t)$ \\
\hline Z-axis & 889 & 33 & 72 & 95 & 60 & 150 & 2 & 0.01 & $5 \sin (t)$ \\
\hline
\end{tabular}

The line chart of Figure 17 indicates the response of the SCARA robot both desired and actual positions are presented in blue and red respectively. Both you and $\mathrm{x}$-axes are presented in the unit meter. In all conclusions from a first glance can see the motions between the two are precisely identical, which illustrates the optimum response for the full ellipse trajectory. The power of the footprint, the ability of the type two fuzzy controllers to deal with systematic uncertainties. Where the action of the impedance control strategy based on type two fuzzy controllers showed a high percentage of error enhancement that can track the root trajectory inaccurate form.

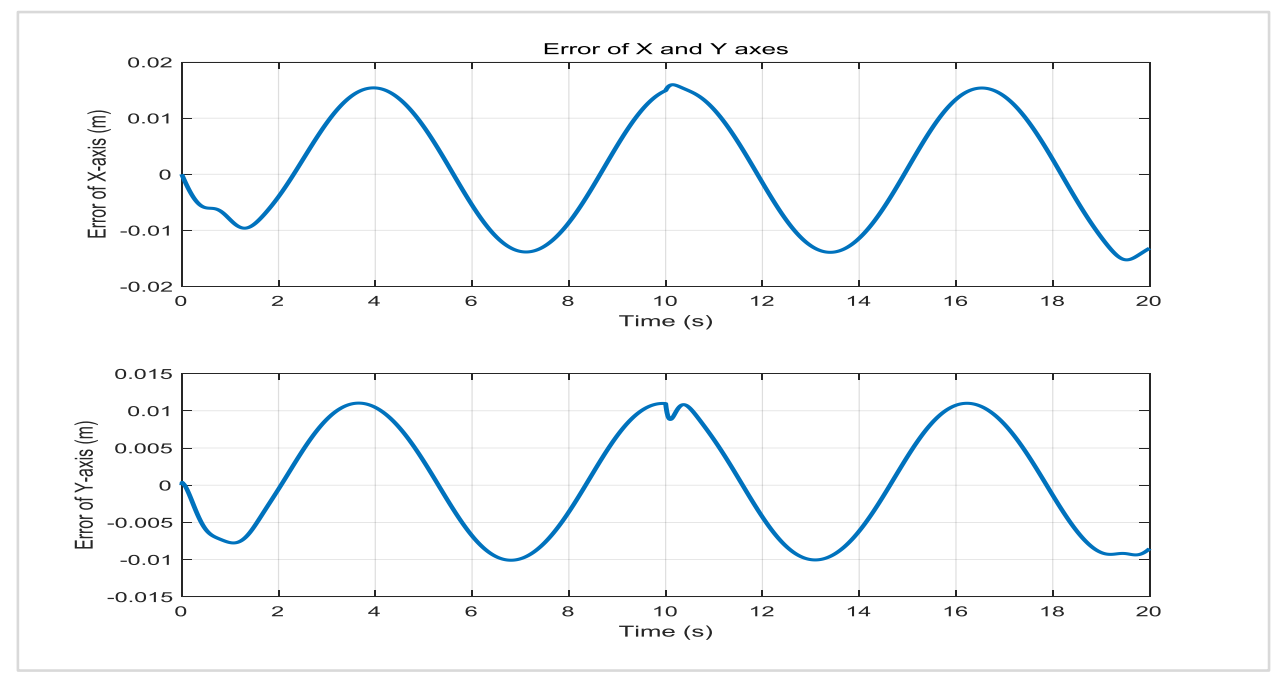

Figure 16 Error signal in the position of the working exes by the half ellipse trajectory 


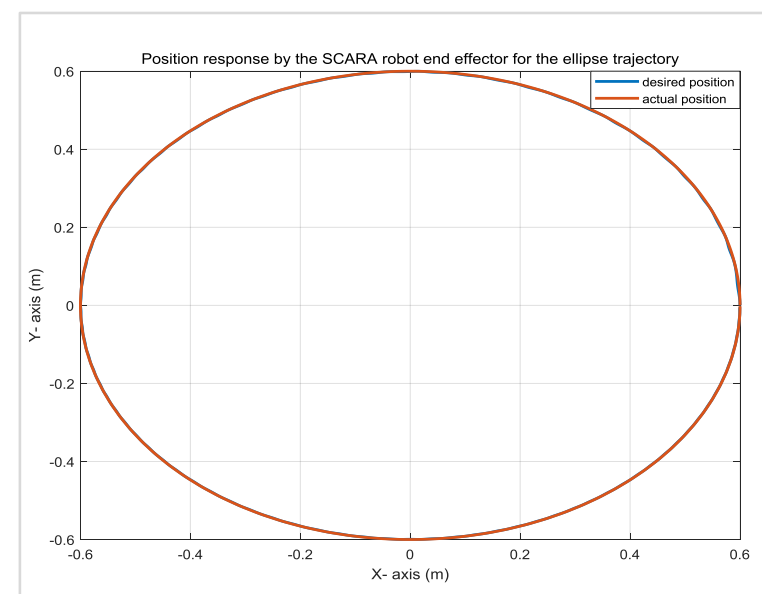

Figure 17 The end effector's position is responsible for the full ellipse trajectory

The line graph of Figure 18 depicts the activity of the torque control signal for three joints $\mathrm{T}_{2}, \mathrm{~T}_{3}, \mathrm{~T}_{4}$ all represented in a blue line. The vertical axis represents torque, which increases every 1.0 in unit NM while the horizontal axis indicates the time, which is presented in a two-second time interval. Both $\mathrm{T}_{2}$ and $\mathrm{T}_{3}$ are similar in pattern change, but differ in quantity, where $T_{2}$ accounts for double $T_{3}$ quantity. However, $\mathrm{T}_{4}$ is independent in both patterns in quantity. Furthermore, $\mathrm{T}_{4}$ holds the lowest degrees with the least fluctuation during the given period. Furthermore, the $T_{2}$ signal varies sinusoidally between the period of 0 to 20 seconds, although in a nonuniform amplitude. The same applies to $\mathrm{T}_{3}$, but the quantity of the signals shortens around $25 \%$ from $\mathrm{T}_{2}$. However, $\mathrm{T}_{4}$ indicates a variation in signals for the first second of the total period. Shortly after returning to its origin and remains at a steady rate of $0 \mathrm{Nm}$.
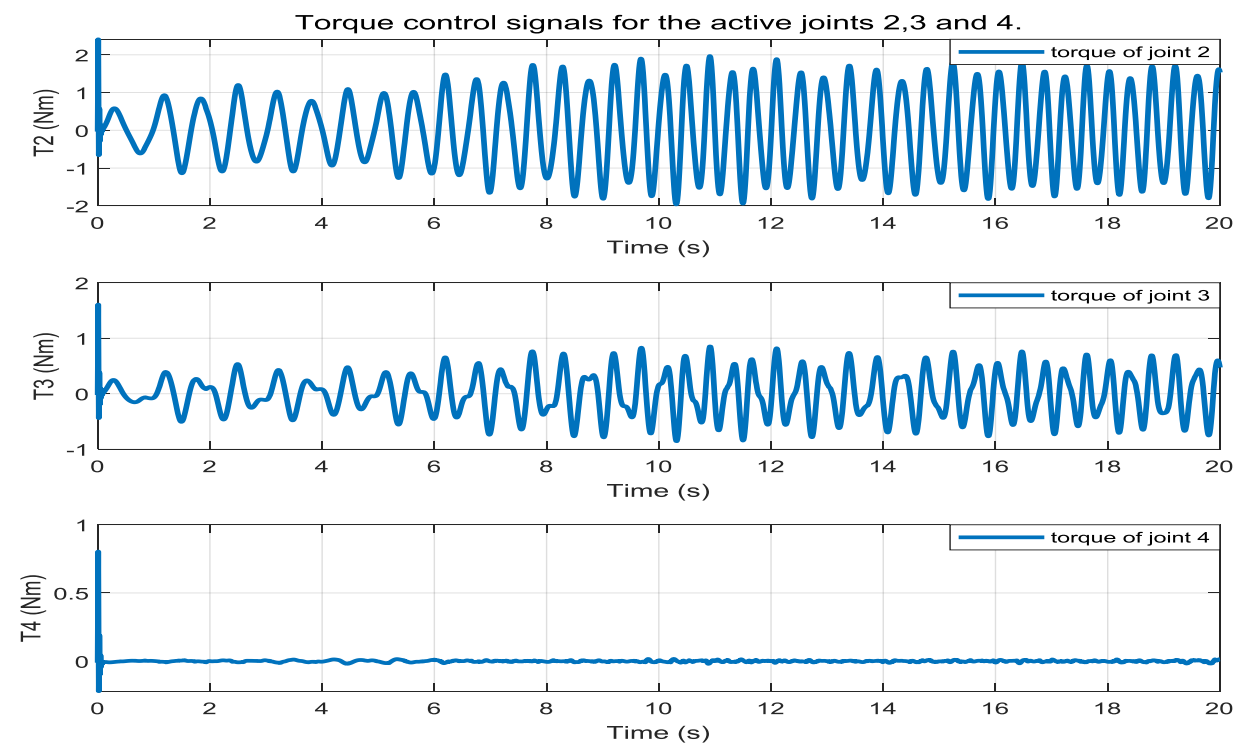

Figure 18 The generated torque control signal in each active joint

The line chart of Figure 19 illustrates three stages of theta responses in three joint angles theta, 2, theta 3, and theta 4 . The blue line represents the activity of the joints, and the vertical axis represents theta inunit degrees, while the horizontal axis indicates the time in unit seconds. Figure 19 indicates the drastic changes in all three curves. Cannot dispute that both theta 3 and theta 4 are almost similar in pattern change but differ in magnitude. However, theta 2 is independent compared to both theta 3 and theta 4 patterns in magnitude. Furthermore, $\mathrm{T}_{2}$ is monotonically increasing that holds the highest degrees with the least fluctuation along the given period. 
Shahad S. Ghintab et al.
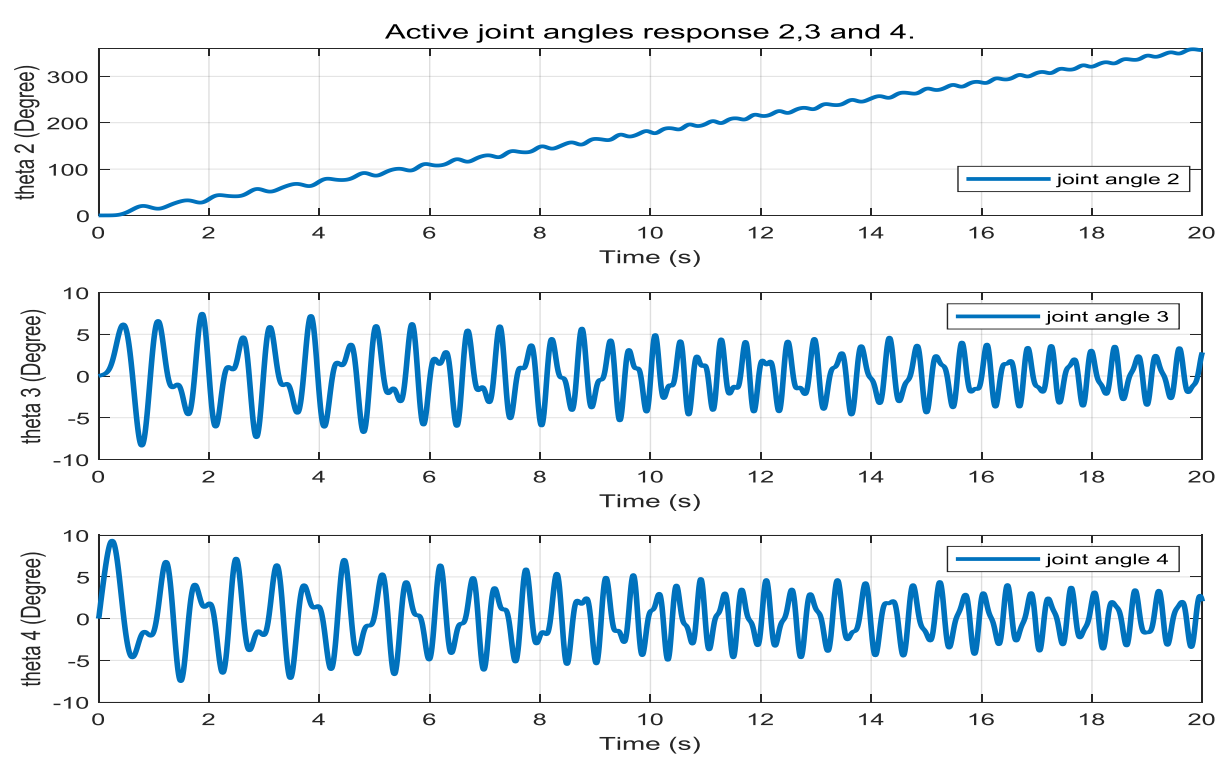

Figure 19 Joint angle response for the active joints

The two-line graphs of Figure 20 depict the error signals that indicate positions of working axes by full ellipse trajectories. Where the overall error rate generated by impedance method-based fuzzy type two PID controllers are nearly $0.004 \mathrm{~m}$ and its optimized for the trajectory tracking process.
Nonetheless, the $\mathrm{x}$-axis shows an increase in position from the very beginning reaches 2 meters in roughly 0.003 seconds. However, begins to decrease in a very unstable motion every 0.25 seconds, reaching minimums -0.02 meters marked at a time of just past 6 seconds.
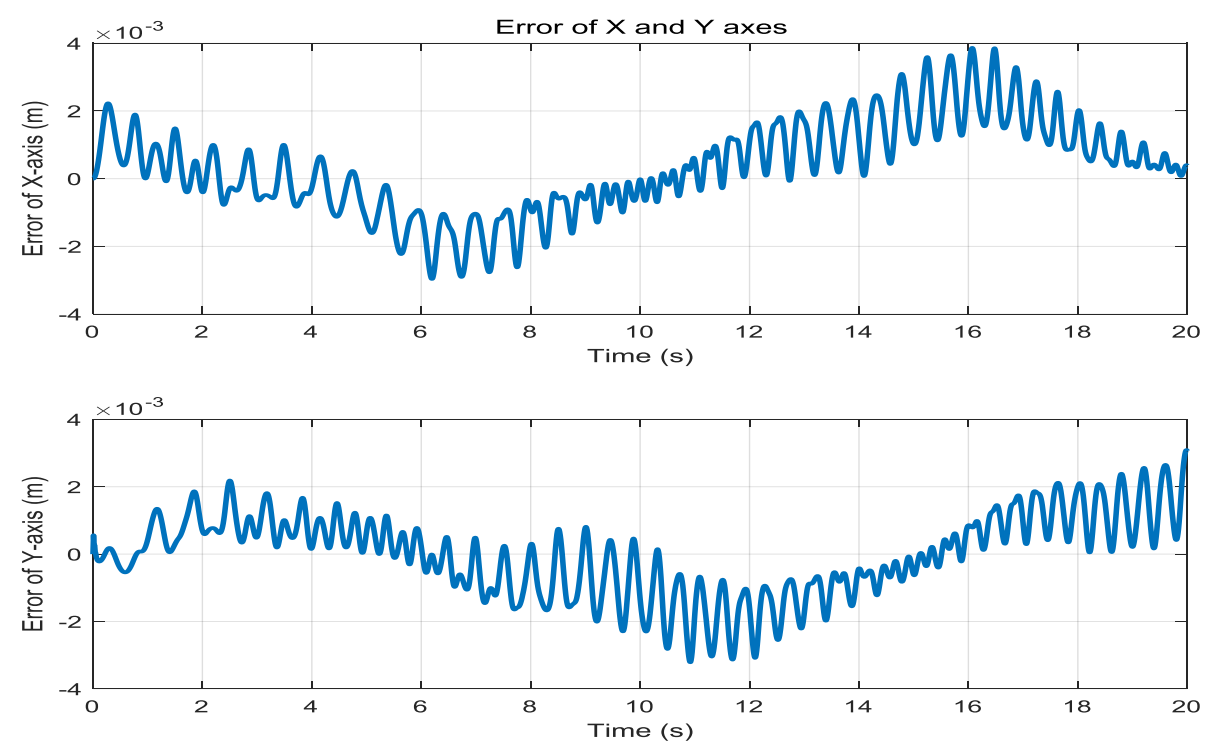

Figure 20 position Error signal of the working axes by the full ellipse trajectory 


\section{Discussion}

Figure 17 Manifests the performance optimality of the applied controller for the SCARA using a fully tracking trajectory compared to Figure 9. Where use impedance method incorporates the type-2 fuzzy PID controller shows results presented in Figures 18, 19, and 20, which compared to the results of the impedance method incorporate FLC type-1 PD controller with Figures 10 and 11 and 12. In detail; Figure 18 shows the optimum actuator torque regulation at the maximum limit of $\pm 1.8 \mathrm{Nm}$ by the type-2 fuzzy PID controller. Figure 19 shows the angles response in each joint motor with maximum utilization. Figure 20 graphs the error signal founded by the FLC type-2 PID controller while regulating the robot end-effector at the full ellipse trajectory with maximum limits of $0.028 \mathrm{~m}$ while the type- 1 fuzzy PD controller gives a larger error.

All gathered results of the proposed controllers have been compared to existing related work [3] as given in Table 7. The main comparison factors are the SCARA robot end-effector position error and oscillation minimization.

The previous works also depend on the same factors gathered by the following controllers: developed sliding mode controller (A), Linear, quadratic regulator learning method $(\mathrm{B})$, and model reference adaptive controller $(\mathrm{C})$, where each one of them will compare with the proposed impedance method that incorporates type-1 fuzzy PD and type-2 PID controllers. The impedance control strategy for regulating the force and position has been developed to the novelty of AI by incorporating the fuzzy system as position controller. Where it efficiently deals with the external disturbances and forces that are applied to the SCARA robot end effector. Especially, type -2 fuzzy PID controllers efficiently deal with the system uncertainties and dynamic errors by the power of the third dimension of the membership function.

The proposed impedance controllers have been tested under the load of the external forces, that applied on the end effector in weight of $1 \mathrm{Nm}$ and $5 \mathrm{Nm}$ with PD and PID controllers respectively. The results have been demonstrated the ability of the proposed control algorithms to deal with these applied disturbances while the presence of variable load uncertainty affects each robot link. The proposed controllers lead to enable the robot from tracking the desired motion trajectories in acceptable ranges and overcome these uncertainties through these measuring factors shown in Table 7. A complete list of abbreviations is shown in Appendix I.

Table 7 The enhanced controlled system characteristics for all proposed controllers compared to [3]

\begin{tabular}{|c|c|c|c|c|}
\hline $\begin{array}{l}\text { The eri } \\
\text { [3]. (A) }\end{array}$ & $\begin{array}{r}\text { ng mode controller The } \\
\text { controll }\end{array}$ & $\begin{array}{c}\text { FLC } \\
\text { d traject }\end{array}$ & Percenta & ncement \\
\hline $\begin{array}{l}\text { X-axis } \\
(\mathbf{m})\end{array}$ & $\begin{array}{ll}\text { Y-axis } & \text { X-axis } \\
(\mathrm{m}) & (\mathrm{m})\end{array}$ & $\begin{array}{l}\text { Y-axis } \\
(\mathbf{m})\end{array}$ & $\begin{array}{l}\text { X-axis } \\
(\mathbf{m})\end{array}$ & $\begin{array}{l}\text { Y-axis } \\
(\mathbf{m})\end{array}$ \\
\hline 0.6 & 0.016 & & FLC type & (A) \\
\hline The er & controller with the 0.041 & 0.012 & $93.166 \%$ & $25 \%$ \\
\hline Learni & [3]. (B) & & FLC type & (B) \\
\hline The el & The error of the & & $92.807 \%$ & $0 \%$ \\
\hline $\mathrm{X}$-axis & Y-axis (m) The er & the FLC & FLC type & (C) \\
\hline & & & $93.278 \%$ & $33.333 \%$ \\
\hline 0.57 & 0.12 & & FLC type & (A) \\
\hline The eri & adaptive controller $X$-axis & Y-axis & $95.501 \%$ & $31.250 \%$ \\
\hline [3]. (C) & (m) & (m) & FLC type & (B) \\
\hline The e & The error of $\mathrm{Y}$ - & & $95.263 \%$ & $90.833 \%$ \\
\hline & axis (m) & 0.011 & FLC type & (C) \\
\hline 0.61 & 0.018 & & $95.574 \%$ & $38.887 \%$ \\
\hline
\end{tabular}

\section{Conclusion and future work}

SCARA redundant robot of 5DoF's nonlinear model has been mathematically modeled under uncertain applied forces to its end effectors and load version in links' weight with friction effect in each joint. The impedance control method in the form of a force position control structure has been developed to deal with system position through tracking the desired design trajectories, the position part designed in two fuzzy architectures, fuzzy logic controller type one 
PD and type two PID. These controllers have been efficiently dealt with robot nonlinear model and friction rejection, with superiority in performance with FLC type-2 PID due to the third-dimension feasibility, while the presence of the external force's disturbances applied to the robot end-effector. The test results of the two developed controllers manifested a maximum enhancement by comparison with the existing related works by the position responses.

As a limitation of this work, the trajectory tracking process cannot be applicable undersupplying external force effect in more than $7 \mathrm{Nm}$.

The future work will be on refining the impedance controller parameters by developing a parallel AI optimizer that can reach the exact motion trajectory by $100 \%$ based on the specially designed objective error minimization function.

\section{Acknowledgment}

None.

\section{Conflicts of interest}

The authors have no conflicts of interest to declare.

\section{Authors' Contribution Statement}

Shahad S. Ghintab: Conceptualization, investigation, data curation, writing - original draft, writing - review and editing. Zeyad A. Karam: Data collection, conceptualization, writing - original draft, analysis and interpretation of results. Sami Hasan: Study conception, design, data collection, supervision, investigation on challenges and draft manuscript preparation.

\section{References}

[1] Kim JO, Khosla PK. Design of space shuttle tile servicing robot: an application of task based kinematic design. In ICRA1993 (pp. 867-74).

[2] Medjebouri A, Mehennaoui L. Active disturbance rejection control of a SCARA robot arm. International Journal of u-and e-Service, Science and Technology. 2015; 8(1):435-46.

[3] Urrea C, Kern J. Trajectory tracking control of a real redundant manipulator of the SCARA type. Journal of Electrical Engineering and Technology. 2016; 11(1):215-26.

[4] Tao W, Xingsong W. Fault diagnosis of a SCARA robot. In international conference on mechatronics and machine vision in practice 2008 (pp. 352-6). IEEE.

[5] Fang J, Li W. Four degrees of freedom SCARA robot kinematics modeling and simulation analysis. International Journal of Computer, Consumer and Control. 2013; 2(4):20-7.

[6] Shariatee M, Akbarzadeh A, Mousavi A, Alimardani S. Design of an economical SCARA robot for industrial applications. In second RSI/ISM international conference on robotics and mechatronics 2014(pp. 534-9). IEEE.

[7] Amiri-m AA, Gharib M, Moavenian M, Torabiz K. Modelling and control of a SCARA robot using quantitative feedback theory. Proceedings of the Institution of Mechanical Engineers, Part I: Journal of Systems and Control Engineering. 2009; 223(7):91928.

[8] Ghintab SS, Karam ZA, Hasan S. Highly-error enhanced smartly-algorithmic structured impedance fuzzy controllers for a SCARA redundant manipulator. Solid State Technology. 2020; 63(6):1727-46.

[9] Adelhedi F, Jribi A, Bouteraa Y, Derbel N. Adaptive sliding mode control design of a SCARA robot manipulator system under parametric variations. Journal of Engineering Science and Technology Review. 2015; 8(5):117-23.

[10] Jaiswal NK, Kumar V. 3-DOF scara type robot manipulator using mamdani based fuzzy controller. International Journal of Scientific Research Engineering \& Technology. 2014; 3(3): 659-63.

[11] Manceur M, Essounbouli N, Hamzaoui A. Secondorder sliding fuzzy interval type-2 control for an uncertain system with real application. IEEE Transactions on Fuzzy Systems. 2011; 20(2):262-75.

[12] Karam ZA, Abdulkadhim NM. Design, implementation, interfacing and control of internet of robot things for human upper limb. International Journal of Computing and Digital System. 2021; 11(1):387-99.

[13] Lian Z, Shi P, Lim CC. Hybrid-triggered interval type2 fuzzy control for networked systems under attacks. Information Sciences. 2021; 567:332-47.

[14] Karam ZA. Pi-like fuzzy logic position controller design for electro-hydraulic servo-actuator based on particle swarm optimization and artificial bee colony algorithms. Al-Nahrain Journal for Engineering Sciences. 2016; 19(2):395-406.

[15] Hou S, Chu Y, Fei J. Adaptive type-2 fuzzy neural network inherited terminal sliding mode control for power quality improvement. IEEE Transactions on Industrial Informatics. 2021; 17(11):7564-74.

[16] Karam ZA, Awad OA. Design of active fractional PID controller based on whale's optimization algorithm for stabilizing a quarter vehicle suspension system. Periodica Polytechnica Electrical Engineering and Computer Science. 2020; 64(3):247-63.

[17] Soriano LA, Rubio JD, Orozco E, Cordova DA, Ochoa G, Balcazar R, et al. Optimization of sliding mode control to save energy in a SCARA robot. Mathematics. 2021; 9(24):1-16.

[18] Zhen S, Ma M, Liu X, Chen F, Zhao H, Chen YH. Model-based robust control design and experimental validation of SCARA robot system with uncertainty. Journal of Vibration and Control. 2022.

[19] Nair S, Elias B, Naidu VP. Image fusion using type-2 fuzzy systems. International Journal of Advanced 
Research in Electrical, Electronics and Instrumentation Engineering. 2014; 3(5):491-504.

[20] Maouche D, Eker I. Adaptive fuzzy type-2 in control of 2-DOF helicopter. International Journal of Electronics and Electrical Engineering. 2017; 5(2):99105.

[21] Karam ZA, Ghintab SS, Hasan S. Rapidlyimplementable optimizely-sizable fuzzy controller architectures: a performance analysis for semiconductor packaging two axes table. Periodicals of Engineering and Natural Sciences. 2019; 7(2):64459

[22] Precup RE, David RC, Roman RC, Szedlak-stinean AI, Petriu EM. Optimal tuning of interval type-2 fuzzy controllers for nonlinear servo systems using slime mould algorithm. International Journal of Systems Science. 2021:1-6.

[23] Hassan MY, Karam ZA. Force-position controller design for 4 dof's rehabilitation robot arm using PSO algorithm. International Journal of Information Technology and Business Management. 2015; 39(1):84-98.

[24] Mohammadi MH, Mohammadzadeh A, Hadjiaghaie VR, Tavoosi J, Khooban MH. A type-2 fuzzy control for active/reactive power control and energy storage management. Transactions of the Institute of Measurement and Control. 2022; 44(5):1014-28.

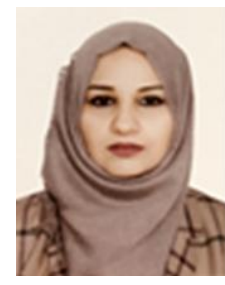

Shahad S. Ghintab received her B.Sc. Degree in Control and Systems Engineering from University of Technology, Iraq, M.Sc. Degree in Control Engineering from University of Technology, Iraq. Currently, she is working as an Assistant Lecturer in the Department of Communication Engineering, AL-Mansour University College Baghdad, Iraq. She gets a certificate in Cisco ccna1 His research area includes Robot Dynamic, Nonlinear systems, and controllers, intelligent optimization methods, intelligent systems, and controllers.

Email: shahad.ghintab@muc.edu.iq

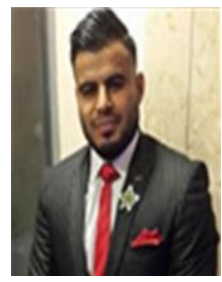

Zeyad A. Karam received his B.Sc. in Control Engineering, graduated from the University of Technology, Iraq, then, M.Sc. In Mechatronics Engineering graduate of University of Technology, Iraq. Currently, working as a board Lecturer for the College of Information Engineering, Al- Nahrain University, Iraq. With 7 years of experience in teaching with three universities, and had a publication of fifteen specialist articles in National and International journals. His working area includes Nonlinear systems control, Robot dynamic, and control, intelligent optimization techniques, intelligent systems design, and control., he is a member of the Iraqi Engineers Syndicate, and a member of the Iraqi Academic Union.

Email: zyad.karam@nahrainuniv.edu.iq

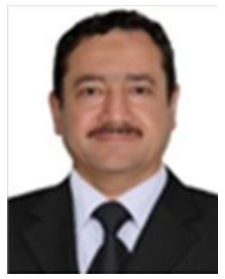

Sami Hasan AI / DSP Architect (Eng. Ph.D.) Newcastle upon Tyne, England, United Kingdom with teaching experience of 25 years in three universities, and he has published many research articles in National and International journals. His research area includes Robot Dynamic, Nonlinear systems, AI controllers, intelligent optimization methods, intelligent systems, and AI architecture.

Email: hhksami@yahoo.com

\begin{tabular}{lll}
\multicolumn{2}{l}{ Appendix I } \\
\hline S. No. & Abbreviation & Description \\
\hline 1 & AI & Artificial Intelligent \\
\hline 2 & DoF & Degree of Freedom \\
\hline 3 & FANN & Fuzzy Artificial Neural Network \\
\hline 4 & FLC & Fuzzy Logic Controller \\
\hline 5 & FOPID & Frictional Order PID \\
\hline 6 & Four & Footprint of Uncertainty \\
\hline 7 & IT2FLC & Interval Type 2 FLC \\
\hline 8 & LMF & Lower Membership Function \\
\hline 9 & NBUL & Negative Big rate \\
\hline 10 & NMUL & Negative, Medium rare \\
\hline 11 & NSUL & Negative Small rate \\
\hline 12 & PBUL & Positive Big rate \\
\hline 13 & PD & Proportional Derivative \\
\hline 14 & PID & Proportional Integral Derivative \\
\hline 16 & PMUL & Positive Medium rare \\
\hline 17 & PSUL & Positive Small rate \\
\hline 18 & QFT & Quantitative Feedback Theory \\
\hline 19 & RMS & Remote Manipulator System \\
\hline 20 & SCARA & Selective Compliance Assembly \\
\hline 21 & SMC & Robot Arm \\
\hline 22 & PD & Type-2 Fuzzy Mode Controller \\
\hline 23 & PID & Type one Fuzzy PD controller \\
\hline 24 & ZMF & Type two Fuzzy PID controller \\
\hline & & Upper Membership Function \\
\hline & Zero rates \\
\hline 15 & \\
\hline 12 &
\end{tabular}

